\title{
Global stabilisation of nonlinear delay systems with a compact absorbing set
}

\author{
Iasson Karafyllis ${ }^{\mathrm{a}, *}$, Miroslav Krstic ${ }^{\mathrm{b}}$, Tarek Ahmed-Ali ${ }^{\mathrm{c}}$ and Francoise Lamnabhi-Lagarrigue ${ }^{\mathrm{d}}$ \\ ${ }^{a}$ Department of Mathematics, National Technical University of Athens, Zografou Campus, 15780 Athens, Greece; ${ }^{b}$ Department of \\ Mechanical and Aerospace Engineering, University of California, San Diego, La Jolla, CA 92093-0411, USA.; ${ }^{c}$ Laboratoire GREYC \\ CNRS-ENSICAEN, 06 Boulevard du Marechal Juin, 14050 Caen Cedex, France; ${ }^{d}$ Centre National de la Recherche Scientifique, \\ CNRS-EECI SUPELEC, 3 rue Joliot Curie, 91192 Gif-sur-Yvette, France
}

\begin{abstract}
Predictor-based stabilisation results are provided for nonlinear systems with input delays and a compact absorbing set. The control scheme consists of an inter-sample predictor, a global observer, an approximate predictor, and a nominal controller for the delay-free case. The control scheme is applicable even to the case where the measurement is sampled and possibly delayed. The input and measurement delays can be arbitrarily large but both of them must be constant and accurately known. The closed-loop system is shown to have the properties of global asymptotic stability and exponential convergence in the disturbance-free case, robustness with respect to perturbations of the sampling schedule, and robustness with respect to measurement errors. In contrast to existing predictor feedback laws, the proposed control scheme utilises an approximate predictor of a dynamic type that is expressed by a system described by integral delay equations. Additional results are provided for systems that can be transformed to systems with a compact absorbing set by means of a preliminary predictor feedback.
\end{abstract}

Keywords: nonlinear systems; predictor feedback; delay systems

\section{Introduction}

Remarkable progress has been made in recent years on the design of predictor feedback laws for nonlinear delay systems (Bekiaris-Liberis \& Krstic, 2012; Bekiaris-Liberis \& Krstic, 2013a, 2013b; Karafyllis, 2011; Karafyllis \& Jiang, 2011; Karafyllis \& Krstic, 2012a, 2013a, XXXXb; Krstic, $2004,2008,2009,2010)$. The main challenge to the implementation and design of predictor feedback for nonlinear delay systems is that, except for rare special cases, the solution mapping (used for the prediction) is not available explicitly.

The current status in the literature on input delay compensation is that when, in addition to input delays,

- the full state is not measured,

- the measurement is sampled and possibly delayed,

and when, in addition to global stability, the following properties are required in closed loop,

- exponential convergence for the disturbance-free case,

- robustness with respect to perturbations of the sampling schedule, and

- robustness with respect to measurement errors,

predictor feedback designs are available only for two classes of systems: linear detectable and stabilisable systems and globally Lipschitz systems in strict feedback form (Karafyllis \& Krstic, 2013a).

In this paper, we present a result that removes the global Lipschitz restriction (an algebraic condition on the system's right-hand side), but imposes an assumption that the system has a compact absorbing set (a condition on the system's dynamic behaviour in open loop). Specifically, we consider general nonlinear systems of the form

$$
\dot{x}(t)=f(x(t), u(t-\tau)), x \in \Re^{n}, u \in U,
$$

where $U \subseteq \Re^{m}$ is a non-empty compact set with $0 \in U$, $\tau \geq 0$ is the input delay and $f: \Re^{n} \times \Re^{m} \rightarrow \Re^{n}$ is a smooth vector field with $f(0,0)=0$. The measurements are sampled and the output is given by

$$
y\left(\tau_{i}\right)=h\left(x\left(\tau_{i}-r\right)\right)+e\left(\tau_{i}\right)
$$

where $h: \Re^{n} \rightarrow \Re^{k}$ is a smooth mapping with $h(0)=0$, $r \geq 0$ is the measurement delay, $\left\{\tau_{i}\right\}_{i=0}^{\infty}$ is a partition of $\Re_{+}$ (the set of sampling times) and the input $e: \Re_{+} \rightarrow \Re$ is the measurement error. We focus on a class of nonlinear systems that is different from the class of globally Lipschitz systems: the systems with a compact absorbing set. A nonlinear system with a compact absorbing set is a system for which all solutions enter a specific compact set after an initial transient period (for systems without inputs

\footnotetext{
*Corresponding author. Email: iasonkar@central.ntua.gr
} 
the name 'global uniform ultimate boundedness' is used in Khalil (1996); the term 'dissipative system' is used in the literature of finite-dimensional dynamical systems; see Stuart and Humphries (1998) and the discussion on page 22 of the book Temam (1997)).

Though it may appear that we merely trade one major restriction (global Lipschitzness) for another (compact absorbing set), which imposes a strong requirement on the system's open-loop behaviour, the latter restriction is less frequently violated in applications. Many engineering systems belong to the class of systems with a compact absorbing set because finite escape is rare in physical processes, control inputs usually saturate, and limit cycles are a frequent outcome of local instabilities.

The contribution of our paper is twofold:

(1) Predictor feedback is designed and stability is proved for the class of nonlinear delay systems with a compact absorbing set under appropriate assumptions (Theorem 2.2).

(2) The result is then extended to nonlinear delay systems that can be transformed to systems with a compact absorbing set by means of a preliminary predictor feedback (Theorem 2.4).

In both cases, we provide explicit formulae for the predictor feedback and explicit inequalities for the parameters of the applied control scheme and the upper diameter of the sampling partition. The proposed predictor feedback guarantees all properties listed at the beginning of the section for the class of nonlinear delay systems with a compact absorbing set: global asymptotic stability and global exponential attractivity in the absence of measurement error, robustness with respect to perturbations of the sampling schedule and robustness with respect to measurement errors.

Our predictor feedback design consists of the following elements:

(1) An Inter-sample predictor (ISP), which uses the sampled, delayed and corrupted measurements of the output and provides an estimate of the (unavailable) delayed continuous output signal.

(2) A global observer $(\mathrm{O})$, which uses the estimate of the delayed continuous output signal and provides an estimate of the delayed state vector.

(3) An approximate or exact predictor (P), which uses the estimate of the delayed state vector in order to provide an estimate of the future state vector.

(4) A delay-free controller (DFC), that is, a baseline feedback law that works for the delay-free version of the system, which in the presence of delay uses the estimate of the future state vector in order to provide the control action.
We refer to the above control scheme as the ISP-O-PDFC control scheme. In Karafyllis and Krstic (2013a), the ISP-O-P-DFC control scheme was shown to achieve all the objectives mentioned at the beginning of this section by using approximate predictors that are based on successive approximations of the solution map for linear detectable and stabilisable systems and globally Lipschitz systems in strict feedback form. Here, we show that the ISP-O-P-DFC control scheme guarantees all the objectives listed at the beginning of this section using dynamic approximate predictors for systems with a compact absorbing set.

This methodological difference relative to Karafyllis and Krstic (2013a) merits further emphasis. We employ here a class of approximate predictors that are implemented by means of a dynamical system: the approximate predictor is a system described by integral delay equations (IDEs; see Karafyllis \& Krstic, 2013b) and consists of a series connection of $N$ approximate predictors (each making a prediction for the state vector $\delta=\frac{r+\tau}{N}$ time units ahead). Such dynamic predictors were introduced in Ahmed-Ali, Karafyllis, and Lamnabhi-Lagarrigue (2013) and Germani, Manes, and Pepe (2002), but here the predictor is designed in a novel way so that the prediction takes values in an appropriate compact set after an initial transient period. The dynamic predictor is different from other predictors proposed in the literature (e.g., exact predictors in Karafyllis and Krstic (2012a) and Krstic (2010); approximate predictors based on successive approximations in Karafyllis (2011) and Karafyllis and Krstic (2013a); and approximate predictors based on numerical schemes in Karafyllis and Krstic (2012b)). Theorem 2.4 employs a novel combination of approximate predictors and exact predictors in the control scheme, which can be used for other classes of nonlinear delay systems.

The main advantage of the dynamic predictor employed here over other predictor approximations (numerical in Karafyllis and Krstic (2012b) or successive approximations in Karafyllis (2011) and Karafyllis and Krstic (2013a)) is the existence of simple formulas (provided in Ahmed-Ali et al. 2013), for the estimation of the asymptotic gain of the measurement error for certain classes of systems. In contrast, the predictor for which the effect of measurement errors is most difficult to quantify is the numerical predictor (Karafyllis \& Krstic, 2012b).

On the other hand, the disadvantages of the dynamic predictor are the difficulty of implementation (one has to approximate numerically the solution of the IDEs or the equivalent distributed delay differential equations) and that it works only for certain classes of nonlinear systems (globally Lipschitz systems and systems with a compact absorbing set). In contrast, the most easily programmable predictor is the numerical predictor (Karafyllis \& Krstic, 2012 b), which is the crudest version of the predictor based on successive approximations (Karafyllis, 2011; Karafyllis \& Krstic, 2013a) - when only one successive approximation 
is used (and many grid points), then the predictor based on successive approximations coincides with the numerical predictor.

Though our approach to stabilisation of nonlinear systems with actuation and measurement delays is based on delay compensation via predictor design - an approach known for its ability to recover nominal performance in the absence of delay and after finite time in the presence of delay - this is not the only option for stabilisation of nonlinear systems with large dead times. For certain classes of nonlinear systems, other approaches exist that are capable of guaranteeing stability and robustness (Mazenc, Malisoff, \& Lin, 2008; Mazenc, Mondie, \& Francisco, 2004).

The paper is structured as follows. Section 2 contains the assumptions and the statements of the main results. The proofs are given in Section 3. Section 4 presents two illustrative examples. Concluding remarks are provided in Section 5.

Notation. Throughout this paper, we adopt the following notation:

- $\Re_{+}:=[0,+\infty)$. A partition of $\Re_{+}$is an increasing sequence $\left\{\tau_{i}\right\}_{i=0}^{\infty}$ with $\tau_{0}=0$ and $\lim _{i \rightarrow \infty} \tau_{i}=+\infty$.

- By $C^{0}(A ; \Omega)$, we denote the class of continuous functions on $A \subseteq \Re^{n}$, which take values in $\Omega \subseteq \Re^{m}$. By $C^{k}(A ; \Omega)$, where $k \geq 1$ is an integer, we denote the class of functions on $A \subseteq \Re^{n}$ with continuous derivatives of order $k$, which take values in $\Omega \subseteq \Re^{m}$.

- By int $(A)$, we denote the interior of the set $A \subseteq \Re^{n}$.

- For a vector $x \in \Re^{n}$, we denote by $x^{\prime}$ its transpose and by $|x|$ its Euclidean norm. $A^{\prime} \in \mathfrak{R}^{n \times m}$ denotes the transpose of the matrix $A \in \mathfrak{R}^{m \times n}$ and $|A|$ denotes the induced norm of the matrix $A \in \mathfrak{R}^{m \times n}$, i.e., $|A|=$ $\sup \left\{|A x|: x \in \Re^{m},|x|=1\right\}$.

- A function $V: \Re^{n} \rightarrow \Re_{+}$is called positive definite if $V(0)=0$ and $V(x)>0$ for all $x \neq 0$. A function $V: \mathfrak{R}^{n} \rightarrow \mathfrak{R}_{+}$is called radially unbounded if the sets $\left\{x \in \mathfrak{R}^{n}: V(x) \leq M\right\}$ are either empty or bounded for all $M \geq 0$.

- For a function $V \in C^{1}(A ; \Re)$, the gradient of $V$ at $x \in A \subseteq \Re^{n}$, denoted by $\nabla V(x)$, is the row vector $\nabla V(x)=\left[\frac{\partial V}{\partial x_{1}}(x) \ldots \frac{\partial V}{\partial x_{n}}(x)\right]$.

- The class of functions $K_{\infty}$ is the class of strictly increasing, continuous functions $a: \Re_{+} \rightarrow \Re_{+}$with $a(0)=0$ and $\lim _{s \rightarrow+\infty} a(s)=+\infty$.

\section{Systems with an absorbing compact set}

Consider the system (1.1) and (1.2). Our main assumption guarantees that there exists a compact set which is robustly globally asymptotically stable (the adjective robust means uniformity to all measurable and essentially bounded inputs $\left.u: \Re_{+} \rightarrow U\right)$. We call the compact set "absorbing” because the solution "is absorbed" in the set after an initial transient period.

H1: There exist a radially unbounded (but not necessarily positive definite) function $V \in C^{2}\left(\Re^{n} ; \Re_{+}\right)$, a positive definite function $W \in C^{1}\left(\Re^{n} ; \Re_{+}\right)$and a constant $R>0$ such that the following inequality holds for all $(x, u) \in \mathfrak{R}^{n} \times U$ with $V(x) \geq R$

$$
\nabla V(x) f(x, u) \leq-W(x)
$$

Indeed, assumption $\mathrm{H} 1$ guarantees that for every initial condition $x(0) \in \Re^{n}$ and for every measurable and essentially bounded input $u: \Re_{+} \rightarrow U$ the solution $x(t)$ of (1.1) enters the compact set $S=\left\{x \in \Re^{n}: V(x) \leq R\right\}$ after a finite transient period, i.e., there exists $T \in C^{0}\left(\Re^{n} ; \Re_{+}\right)$such that $x(t) \in S$, for all $t \geq T(x(0))$. Moreover, notice that the compact set $S=\left\{x \in \Re^{n}: V(x) \leq R\right\}$ is positively invariant. This fact is guaranteed by the following lemma that is an extension of Theorem 5.1 in Khalil (1996, p. 211).

Lemma 2.1: Consider system (1.1) under hypothesis $\mathrm{H} 1$. Then there exists $T \in C^{0}\left(\Re^{n} ; \Re_{+}\right)$such that for every $x_{0} \in$ $\mathfrak{R}^{n}$ and for every measurable and essentially bounded input $u:[-\tau,+\infty) \rightarrow U$ the solution $x(t) \in \mathfrak{R}^{n}$ of $(1.1)$ with initial condition $x(0)=x_{0}$ and corresponding to input $u$ : $[-\tau,+\infty) \rightarrow U$ satisfies $V(x(t)) \leq \max \left(V\left(x_{0}\right), R\right)$ for all $t \geq 0$ and $V(x(t)) \leq R$ for all $t \geq T\left(x_{0}\right)$.

Our second assumption guarantees that we are in a position to construct an appropriate local exponential stabiliser for the delay-free version system (1.1), i.e. system (1.1) with $\tau=0$.

H2: There exist a positive definite function $P \in$ $C^{2}\left(\Re^{n} ; \Re_{+}\right)$, constants $\mu, K_{1}>0$ with $K_{1}|x|^{2} \leq P(x)$ for all $x \in \Re^{n}$ with $V(x) \leq R$ and a globally Lipschitz mapping $k: \Re^{n} \rightarrow U$ with $k(0)=0$ such that the following inequality holds

$$
\begin{aligned}
& \nabla P(x) f(x, k(x)) \leq-2 \mu|x|^{2}, \\
& \text { for all } x \in \mathfrak{R}^{n} \text { with } V(x) \leq R .
\end{aligned}
$$

The requirement that the mapping $k: \Re^{n} \rightarrow U$ with $k(0)=0$ is globally Lipschitz is not essential. Note that if assumption $\mathrm{H} 2$ holds for certain locally Lipschitz mapping $k: \Re^{n} \rightarrow U$ with $k(0)=0$ and if $U$ is a star-shaped set (i.e. $(\lambda u \in U)$ for every $\lambda \in U[0,1]$ and $u \in U)$, then we are in a position to define $\tilde{k}(x)=$ $(R+1-\min (R+1, \max (R, V(x)))) k(x)$ and we note that assumption $\mathrm{H} 2$ holds for the globally Lipschitz function $\tilde{k}: \Re^{n} \rightarrow U$.

Our third assumption guarantees that we are in a position to construct an appropriate local exponential observer for the delay-free version of system (1.1), (1.2), i.e. systems (1.1) and (1.2) with $r=\tau=0$.

H3: There exist a symmetric and positive definite matrix $Q \in \Re^{n \times n}$, constants $\omega>0, b>R$ and a matrix $L \in \Re^{n \times k}$ 
such that the following inequality holds:

$$
\begin{aligned}
& (z-x)^{\prime} Q(f(z, u)+L(h(z)-h(x))-f(x, u)) \\
& \quad \leq-\omega|z-x|^{2} \\
& \quad \text { for all } u \in U, z, x \in \Re^{n} \text { with } V(z) \leq b \text { and } V(x) \leq R .
\end{aligned}
$$

Indeed, assumption $\mathrm{H} 3$ in conjunction with assumption $\mathrm{H} 1$ guarantees that for every $x(0) \in S=\left\{x \in \Re^{n}: V(x) \leq R\right\}$ and for every measurable and essentially bounded input $u: \Re_{+} \rightarrow U$ the solution of system (1.1), (1.2) with $r=$ $\tau=0, e \equiv 0$ and

$$
\dot{z}=f(z, u)+L(h(z)-y)
$$

satisfies an estimate of the form $|z(t)-x(t)| \leq$ $M \exp (-\sigma t) \quad|z(0)-x(0)|$, for all $t \geq 0$ for appropriate constants $M, \sigma>0$, provided that the initial estimation error $|z(0)-x(0)|$ is sufficiently small. This is why system (2.4) is termed 'a local exponential observer'. The reader should note that assumption H3 holds automatically for nonlinear systems of the form

$$
\begin{aligned}
& \dot{x}_{1}=f_{1}\left(x_{1}\right)+x_{2} \\
& \dot{x}_{2}=f_{2}\left(x_{1}, x_{2}\right)+x_{3} \\
& \vdots \\
& \dot{x}_{n}=f_{n}\left(x_{1}, \ldots, x_{n}\right)+u \\
& y=x_{1}
\end{aligned}
$$

for every $b>R>0$ and for every non-empty set $U \subseteq \Re^{m}$, where $f_{i}: \Re^{i} \rightarrow \Re(i=1, \ldots, n)$ are smooth mappings.

In order to be able to construct a feedback stabiliser for system (1.1) and (1.2), we need an additional technical assumption.

H4: There exist constants $c \in(0,1), R \leq a<b$ such that the following inequality holds:

$$
\begin{aligned}
& \nabla V(z)(f(z, u)+L(h(z)-h(x))) \leq-W(z)+(1-c) \\
& \quad \times|\nabla V(z)|^{2} \\
& \quad \times \frac{(z-x)^{\prime} Q(f(z, u)+L(h(z)-h(x))-f(x, u))}{\nabla V(z) Q(z-x)}
\end{aligned}
$$

for all $u \in U, z, x \in \Re^{n}$ with $a<V(z) \leq b$,

$\nabla V(z) Q(z-x)<0$ and $V x \leq R$.

Assumption $\mathrm{H} 4$ imposes constraints for the evolution of the trajectories of the local observer (2.4). Indeed, inequality (2.6) imposes a bound on the derivative of the Lyapunov function $V \in C^{1}\left(\Re^{n} ; \Re_{+}\right)$along the trajectories of the local observer (2.4) for certain regions of the state space. Assumption $\mathrm{H} 4$, in conjunction with assumptions $\mathrm{H} 1$ and $\mathrm{H} 3$, allows us to construct a global exponential sampled-data observer for system (1.1) and (1.2) in the same spirit as in Ahmed-Ali et al. (2013): first, we exploit assumption H4 to construct an observer for which the observer states enter the compact set $\tilde{S}=\left\{z \in \mathfrak{R}^{n}: V(z) \leq b\right\}$ after some finite time. Since assumption H1 guarantees that the states of the system enter the compact set $S=\left\{x \in \Re^{n}: V(x) \leq R\right\}$ after some finite time, we can guarantee that the system operates on the set for which assumption $\mathrm{H} 3$ holds after an initial transient period. Therefore, we can exploit the local exponential observer of assumption H3 and guarantee exponential convergence of the error after an initial transient period.

We are now ready to state the first main result of the paper. Note that the dynamic feedback stabiliser is explicitly given and that all parameters included in the feedback stabiliser are required to satisfy explicit inequalities that can be verified easily in practice.

Theorem 2.2: $\quad$ Consider systems (1.1) and (1.2) under assumptions $\mathrm{H} 1-\mathrm{H} 4$. Define

$$
\begin{aligned}
& \hat{k}(z, y, u):= L(h(z)-y), \text { for all }(z, y, u) \in \Re^{n} \times \Re^{k} \\
& \times U \text { with } V(z) \leq R, \\
& \hat{k}(z, y, u):= L(h(z)-y)-\frac{\varphi(z, y, u)}{|\nabla V(z)|^{2}}(\nabla V(z))^{\prime}, \\
& \quad \text { for all }(z, y, u) \in \Re^{n} \times \Re^{k} \times U \text { with } V(z)>R,
\end{aligned}
$$

where $\varphi: \Re^{n} \times \Re^{k} \times \Re^{m} \rightarrow \Re_{+}$is defined by

$$
\begin{aligned}
\varphi(z, y, u):= & \max (0, \nabla V(z) f(z, u)+W(z) \\
& +p(V(z)) \nabla V(z) L(h(z)-y))
\end{aligned}
$$

and $p: \Re_{+} \rightarrow[0,1]$ is an arbitrary locally Lipschitz function that satisfies $p(s)=1$ for all $s \geq b$ and $p(s)=0$ for all $s \leq a$. Let $q: \Re \rightarrow \Re_{+}$be a continuously differentiable function with $q(s)=1$ for $s \leq 1$ and $s q(s) \leq K$ for $s \geq 1$, where $K \geq 1$ is a constant. Let $\psi: \Re^{n} \rightarrow[1,+\infty)$ be a smooth function that satisfies the following implication:

$$
V(x) \leq \max (V(z), b) \Rightarrow|x| \leq \psi(z) .
$$

Let $N>0$ be an integer and $T_{s}>0, \sigma>0$ be constants so that

$$
\begin{gathered}
\sigma \leq \min \left(\frac{\mu}{\sqrt{n} \tilde{P}}, \frac{c \omega}{4|Q|}\right), \delta M_{1}^{q} M_{1}^{f} e^{\sigma \delta}<1 \\
\quad \text { and } T_{s} G_{1} e^{\sigma T_{s}} \sqrt{\frac{2|Q|}{K_{2}}} \frac{G_{2}|Q|}{c \omega}<1,
\end{gathered}
$$

where $\quad M_{1}^{q}:=\sup \left\{\frac{\left|q\left(\frac{|\xi|}{\psi(z)}\right) \xi-q\left(\frac{|x|}{\psi(z)}\right) x\right|}{|x-\xi|}: x \in S_{1}, \xi \in S_{3}\right.$, $\left.z \in S_{2}, x \neq \xi\right\}, M_{1}^{f}:=\sup \left\{\frac{|f(x, u)-f(\xi, u)|}{|x-\xi|}: x \in S_{1}, \xi \in S_{4}\right.$, $u \in U, x \neq \xi\}, \quad S_{1}:=\left\{x \in \Re^{n}: V(x) \leq R\right\}, \quad S_{2}:=$ 
$\left\{x \in \Re^{n}: V(x) \leq b\right\}$,

$S_{3}:=\left\{x \in \Re^{n}:|x| \leq K \psi(z)+\delta p(K \psi(z)), z \in S_{2}\right\}$,

$\delta=\frac{r+\tau}{N}, \quad G_{1}:=\sup \left\{\frac{|\nabla h(x) f(x, u)-\nabla h(z) f(z, u)|}{|x-z|}:\right.$ $\left.x \in S_{1}, z\right\} \in S_{2}, u \in U, x \neq z, \quad \tilde{p}(s):=$ $\max \left\{|f(z, u)|:(z, u) \in \Re^{n} \times U,|z| \leq s\right\}, \quad G_{2}:=$ $\sup \left\{\frac{|\hat{k}(z, y, u)-\hat{k}(z, w, u)|}{|y-w|}: y, w \in \mathfrak{R}^{k}, z \in S_{2}, u \in U\right.$,

$y \neq w\}, \quad S_{4}:=\left\{x \in \Re^{n}:|x| \leq K \psi(z), z \in S_{2}\right\}, \quad \tilde{P}:=$ $\max \left\{\left|\nabla^{2} P(x)\right|: x \in \operatorname{co}\left(S_{1}\right)\right\}, \operatorname{co}\left(S_{1}\right)$ denotes the convex hull of $S_{1}$ and $K_{2} \in(0,|Q|]$ is a constant for which the inequality $K_{2}|x|^{2} \leq x^{\prime} Q x$ for all $x \in \Re^{n}$.

Then there exist a constant $\Gamma>0$ and a locally Lipschitz function $C \in K_{\infty}$ such that for every partition $\left\{\tau_{i}\right\}_{i=0}^{\infty}$ of $\Re_{+}$with $\sup _{i \geq 0}\left(\tau_{i+1}-\tau_{i}\right) \leq T_{s}, \quad e \in L^{\infty}\left(\Re_{+} ; \Re^{k}\right)$, $\xi_{i, 0} \in L^{\infty}\left([-\delta, 0) ; \Re^{n}\right) \quad(i=1, \ldots, N), \quad\left(z_{0}, w_{0}\right) \in \Re^{n} \times$ $\Re^{k}, x_{0} \in C^{0}\left([-r, 0] ; \Re^{n}\right), u_{0} \in L^{\infty}([-r-\tau, 0) ; U)$, the solution of (1.1) and (1.2) with

$$
\begin{gathered}
\dot{z}(t)=f(z(t), u(t-r-\tau))+\hat{k}(z(t), w(t), u(t-r-\tau)), \\
\text { for } t \geq 0, \\
\qquad \begin{aligned}
\dot{w}(t) & =\nabla h(z(t)) f(z(t), u(t-r) \tau)), \\
& \text { for } t \in\left[\tau_{i}, \tau_{i+1}\right), i \geq 0,
\end{aligned}
\end{gathered}
$$

$$
w\left(\tau_{i}\right)=y\left(\tau_{i}\right)=h\left(x\left(\tau_{i}-r\right)\right)+e\left(\tau_{i}\right), \text { for } i \geq 1,
$$

$$
\begin{aligned}
\xi_{j}(t)= & q\left(\frac{\left|\xi_{j-1}(t)\right|}{\psi(z(t))}\right) \xi_{j-1}(t)+\int_{0}^{\delta} f\left(q\left(\frac{\left|\xi_{j}(t+s-\delta)\right|}{\psi(z(t))}\right)\right. \\
& \left.\times \xi_{j}(t+s-\delta), u(t+(j-1) \delta-\tau-r+s)\right) d s, \\
& \text { for } t \geq 0, j=1, \ldots, N,
\end{aligned}
$$

where $\delta=\frac{r+\tau}{N}$, with $\xi_{0}(t)=z(t)$ and

$$
u(t)=k\left(\xi_{N}(t)\right), \text { for } t \geq 0
$$

initial condition $\xi_{j}(\theta)=\xi_{j, 0}(\theta)$ for $\theta \in[-\delta, 0) \quad(j=$ $1, \ldots, N), \quad(z(0), w(0))=\left(z_{0}, w_{0}\right), \quad x(\theta)=x_{0}(\theta) \quad$ for $\theta \in[-r, 0], \quad u(\theta)=u_{0}(\theta) \quad$ for $\quad \theta \in[-r-\tau, 0), \quad$ exists and satisfies the following estimate for all $t \geq 0$ :

$$
\begin{aligned}
& \sup _{-r \leq s \leq 0}(|x(t+s)|)+|w(t)|+|z(t)|+ \\
& \sum_{j=1}^{N} \sup _{-\delta \leq s<0}\left(\left|\xi_{j}(t+s)\right|\right)+\sup _{-r-\tau \leq s<0}(|u(t+s)|) \\
& \leq e^{-\sigma t} C\left(\sup _{-r \leq s \leq 0}\left(\left|x_{0}(s)\right|\right)+\left|z_{0}\right|+\left|w_{0}\right|\right. \\
& +\sum_{j=1}^{N} \sup _{-\delta \leq s<0}\left(\left|\xi_{j, 0}(s)\right|\right)+\sup _{-r-\tau \leq s<0}\left(\left|u_{0}(s)\right|\right)
\end{aligned}
$$

$$
\left.+\sup _{0 \leq s \leq t}(|e(s)|)\right)+\Gamma \sup _{0 \leq s \leq t}(|e(s)|) .
$$

\section{Remark 2.3:}

(a) As noted in the Introduction, Theorem 2.2 shows that the control scheme that consists of the series connection of (i) the sampled-data hybrid observer (2.12)-(2.14), which provides an estimate of the delayed state vector $x(t-r)$, (ii) the dynamic predictor (2.15) which provides an estimate of the future value of the state vector $x(t+\tau)$, and (iii) the control law (2.16) is successful under assumptions H1$\mathrm{H} 4$ provided that the upper diameter of the sampling partition $T_{s}>0$ is sufficiently small. The result of Theorem 2.2 guarantees robustness with respect to perturbations of the sampling schedule (inequality (2.17) holds for every sampling partition $\left\{\tau_{i}\right\}_{i=0}^{\infty}$ with upper diameter less or equal to $T_{s}>0$ ).

(b) The sampled-data hybrid observer (2.12)-(2.14) uses the local exponential observer involved in assumptions $\mathrm{H} 3$ and $\mathrm{H} 4$ with some modifications. The first modification involves the replacement of the unavailable output signal $y(t-r)$ with the signal $w(t)$, which is generated by the ISP (2.13) and (2.14) (see also Ahmed-Ali et al., 2013). The second modification is the addition of a 'correction term' of the form $-\frac{\varphi(z, y, u)}{|\nabla V(z)|^{2}}(\nabla V(z))^{\prime}$ which has the task to guarantee the validity of the differential inequality $\nabla V(z)(f(z, u)+\hat{k}(z, y, u)) \leq-W(z)$ for all $(z, y, u) \in \Re^{n} \times \Re^{k} \times U$ with $V(z) \geq b$. The 'correction term' $-\frac{\varphi(z, y, u)}{|\nabla V(z)|^{2}}(\nabla V(z))^{\prime}$ was used in Ahmed-Ali et al. (2013) in order to guarantee that the solution enters an appropriate compact set in finite time and in this appropriate compact set the local exponential observer works.

(c) The input $e: \Re_{+} \rightarrow \Re$ quantifies the effect of measurement errors. Inequality 2.17 shows that the 'asymptotic gain' of the closed-loop system with respect to the measurement error is linear, i.e. $\lim \sup _{t \rightarrow+\infty}\left(\sup _{-r \leq s \leq 0}(|x(t+s)|)+\right.$ $|w(t)|+|z(t)|+\sum_{j=1}^{N} \sup _{-\delta \leq s<0}\left(\left|\xi_{j}(t+s)\right|\right)+$ $\left.\sup _{-r-\tau \leq s<0}(|u(t+s)|)\right) \leq \Gamma \lim \sup _{t \rightarrow+\infty}|e(t)|$. However, the ISS-like inequality (2.17) does not guarantee the ISS property with linear gain. In general, the locally Lipschitz function $C \in K_{\infty}$ is nonlinear and the gain function with respect to the measurement error is nonlinear.

(d) The predictor (2.15) is a system described by IDEs (see Karafyllis \& Krstic, 2013b) and consists of the series connection of $N$ predictors (each making a prediction for the state vector $\delta$ time units ahead). Such dynamic predictors were used in (Ahmed-Ali et al., 2013; Germani et al., 2002) but here the 
predictor (2.15) has an important difference with other predictors: the use of the terms $q\left(\frac{\left|\xi_{j-1}(t)\right|}{\psi(z(t))}\right) \xi_{j-1}(t)$ instead of $\xi_{j-1}(t)$ guarantees that the prediction will take values in an appropriate compact set. The dynamic predictor (2.15) is different from other predictors proposed in the literature (e.g., exact predictors in Karafyllis \& Krstic, 2012a; Krstic, 2010; approximate predictors based on successive approximations in Karafyllis, 2011; Karafyllis \& Krstic, 2013a; approximate predictors based on numerical schemes in Karafyllis \& Krstic, 2012b).

(e) An example of a function $q: \Re \rightarrow \Re_{+}$that satisfies the requirements of Theorem 2.2 is the function $q(s):=2 s^{-1}-s^{-2}$ for $s>1$ and $q(s)=1$ for $s \leq 1$.

(f) Since the function $C \in K_{\infty}$ is a locally Lipschitz function, it follows from estimate (2.17) that the dynamic hybrid controller (2.12)-(2.16) guarantees not only global asymptotic stability but local exponential stability as well in the absence of measurement error. Note that the stability properties of the closed-loop system are robust with respect to perturbations of the sampling schedule.

The following result uses a preliminary predictor feedback in order to transform the given system to a system with a compact absorbing set. However, the result of Theorem 2.4 does not allow us to conclude robust global asymptotic stability for the closed-loop system: only exponential attractivity holds for the closed-loop system. The notion of forward completeness used in the statement of Theorem 2.4 is the standard notion used in Angeli and Sontag (1999): the solution exists for times, all initial conditions and all measurable and locally essentially bounded inputs.

Theorem 2.4: Consider the forward complete system

$$
\begin{aligned}
& \dot{x}(t)=\tilde{f}(x(t), v(t-\tau)), \\
& x(t) \in \mathfrak{R}^{n}, \quad v(t) \in \mathfrak{R}^{m},
\end{aligned}
$$

where $\tau>0$ is the input delay, $\tilde{f}: \Re^{n} \times \Re^{m} \rightarrow \Re^{n}$ is a smooth vector field with $\tilde{f}(0,0)=0$ and sampled measurements given by

$$
y\left(\tau_{i}\right)=h\left(x\left(\tau_{i}-r\right)\right),
$$

where $h: \Re^{n} \rightarrow \Re^{k}$ is a smooth mapping with $h(0)=0$, $r \geq 0$ is the measurement delay and $\left\{\tau_{i}\right\}_{i=0}^{\infty}$ is a partition of $\mathfrak{R}_{+}$(the set of sampling times).

Suppose that there exist smooth functions $a_{1}: \Re^{n} \rightarrow$ $\mathfrak{R}^{l}, a_{2}: \mathfrak{R}^{l} \rightarrow \mathfrak{R}^{m}$ with $a_{1}(0)=0, a_{1}(0)=0$ and a nonempty compact set $U \subset \Re^{m}$ with $0 \in U$ such that the vector field $f(x, u):=\tilde{f}\left(x, a_{2}\left(a_{1}(x)\right)+u\right)$ satisfies assumptions
H1-H4. Moreover, suppose that there exists a locally Lipschitz vector field $g: \mathfrak{R}^{l} \times \mathfrak{R}^{m} \rightarrow \mathfrak{R}^{l}$ with $g(0,0)=0$ such that the equation $\nabla a_{1}(x) \tilde{f}(x, v)=g\left(a_{1}(x), v\right)$ holds for all $(x, v) \in \Re^{n} \times \Re^{m}$. Assume that the system

$$
\begin{aligned}
& \dot{\theta}(t)=g\left(\theta(t), a_{2}(\theta(t))+u(t)\right) \\
& \theta(t) \in \Re^{l}, u(t) \in U
\end{aligned}
$$

is forward complete. Finally, suppose that there exists a mapping $\Phi: \Re^{k} \times L^{\infty}\left([-\tau-r, 0) ; \Re^{m}\right)$ such that for ev$\operatorname{ery}\left(x_{0}, v\right) \in \Re^{n} \times L^{\infty}\left([-\tau,+\infty) ; \Re^{m}\right)$ the solution $x(t) \in$ $\mathfrak{R}^{n}$ of (2.18) with initial condition $x(0)=x_{0}$ corresponding to input $v \in L^{\infty}\left([-\tau,+\infty) ; \mathfrak{R}^{m}\right)$ satisfies for all $t \geq r$ :

$$
a_{1}(x(t+\tau))=\Phi\left(h(x(t-r)), v_{t}\right),
$$

where $\left(v_{t}\right)(s)=v(t+s)$ for $s \in[-\tau-r, 0)$.

Let $\hat{k}: \Re^{n} \times \Re^{k} \times \Re^{m} \rightarrow \Re^{n}$ be the vector field defined by (2.7)-(2.9) for certain locally Lipschitz function $p: \Re_{+} \rightarrow[0,1]$ that satisfies $p(s)=1$ for all $s \geq b$ and $p(s)=0$ for all $s \leq a$. Let $q: \Re \rightarrow \Re_{+}$be a continuously differentiable function with $q(s)=1$ for $s \leq 1$ and $s q(s) \leq K$ for $s \geq 1$, where $K \geq 1$ is a constant. Let $\psi: \Re^{n} \rightarrow[1,+\infty)$ be a smooth function that satisfies implication (2.10). Let $N>0$ be an integer and $T_{s}>0, \sigma>0$ be constants so that (2.11) holds.

Then for every partition $\left\{\tau_{i}\right\}_{i=0}^{\infty}$ of $\Re_{+}$with $\sup _{i \geq 0}\left(\tau_{i+1}-\tau_{i}\right) \leq T_{s}, \quad \xi_{i, 0} \in L^{\infty}\left([-\delta, 0) ; \Re^{n}\right) \quad(i=$ $1, \ldots, N), \quad\left(z_{0}, w_{0}, \theta_{0}\right) \in \mathfrak{R}^{n} \times \mathfrak{R}^{k} \times \mathfrak{R}^{l}, \quad x_{0} \in C^{0}([-r, 0]$; $\left.\Re^{n}\right), \quad u_{0} \in L^{\infty}([-r-\tau, 0) ; U), \quad v_{0} \in L^{\infty}\left([-\tau, 0) ; \Re^{m}\right)$, the solution of (2.18) and (2.19) with (2.12)-(2.16) and

$$
\begin{gathered}
v(t)=u(t)+a_{2}(\theta(t)), \text { for } t \geq 0, \\
\dot{\theta}(t)=g\left(\theta(t), a_{2}(\theta(t))+u(t)\right), \text { for } t \in\left[\tau_{i}, \tau_{i+1}\right), i \geq 0,
\end{gathered}
$$

$$
\theta\left(\tau_{i}\right)=\Phi\left(y\left(\tau_{i}\right), v_{\tau_{i}}\right), \text { for } i \geq 1,
$$

with $\xi_{0}(t)=z(t)$ and initial condition $\xi_{j}(s)=\xi_{j, 0}(s)$ for $s \in[-\delta, 0) \quad(j=1, \ldots, N), \quad(z(0), w(0), \theta(0))=$ $\left(z_{0}, w_{0}, \theta_{0}\right), x(s)=x_{0}(s)$ for $s \in[-r, 0], u(s)=u_{0}(s)$ for $s \in[-r-\tau, 0), v(s)=v_{0}(s)$ for $s \in[-\tau, 0)$ exists for all $t \geq 0$ and satisfies:

$$
\limsup _{t \rightarrow+\infty}\left(e^{\sigma t} P(t)\right)<+\infty
$$

where $\quad P(t):=\sup _{-r \leq s \leq 0}(|x(t+s)|)+|w(t)|+|z(t)|$ $+|\theta(t)|+\sum_{j=1}^{N} \sup _{-\delta \leq s<0}\left(\left|\xi_{j}(t+s)\right|\right)+\sup _{-r-\tau \leq s<0}$ $(|u(t+s)|)$.

Remark 2.5: Theorem 2.4 uses a combination of exact predictors (in the spirit of Karafyllis \& Krstic, 2012a); 
(2.21) is an exact prediction of $\left.a_{1}(x(t+\tau))\right)$ and approximate predictors $((2.15)$ and (2.16) provides an approximate prediction of $k(x(t+\tau)))$. Therefore, Theorem 2.4 generalises the results in Karafyllis and Krstic (2012a) and the result of Theorem 2.2. However, as remarked above the result of Theorem 2.4 is simple exponential attractivity for the closed-loop system. The existence of functions $a_{1}: \mathfrak{R}^{n} \rightarrow \mathfrak{R}^{l}, a_{2}: \mathfrak{R}^{l} \rightarrow \mathfrak{R}^{m}$ satisfying the assumptions of Theorem 2.4 is a restrictive assumption, which can be verified in certain cases (see Example 4.2).

\section{Proofs of main results}

We start with the proof of Theorem 2.2.

Proof of Theorem 2.2: We first notice that the following inequality holds for all $(z, w, u) \in \mathfrak{R}^{n} \times \mathfrak{R}^{k} \times U$ with $V(z) \geq b$ :

$$
\nabla V(z)(f(z, u)+\hat{k}(z, w, u)) \leq-W(z)
$$

Definition (2.8) implies $\nabla V(z)(f(z, u)+\hat{k}(z, w, u))=$ $\nabla V(z)(f(z, u)+L(h(z)-w))-\varphi(z, w, u) . \quad$ Ву distinguishing the cases $\nabla V(z) f(z, u)+W(z)+$ $\nabla V(z) L(h(z)-w) \leq 0 \quad$ and $\quad \nabla V(z) f(z, u)+W(z)+$ $\nabla V(z) L(h(z)-w)>0$, using definition (2.9) and noticing that $p(V(z))=1$, we conclude that (3.1) holds.

Let $\left\{\tau_{i}\right\}_{i=0}^{\infty}$ be a partition of $\Re_{+}$with $\sup _{i \geq 0}\left(\tau_{i+1}-\tau_{i}\right) \leq T_{s}, \quad x_{0} \in C^{0}\left([-r, 0] ; \Re^{n}\right), \quad u_{0} \in L^{\infty}$ $([-r-\tau, 0) ; U), \xi_{i, 0} \in L^{\infty}\left([-\delta, 0) ; \Re^{n}\right)(i=1, \ldots, N)$, $\left(z_{0}, w_{0}\right) \in \Re^{n} \times \mathfrak{R}^{k}, e \in L_{\text {loc }}^{\infty}\left(\Re_{+} ; \Re^{k}\right)$ and consider the solution of (1.1) and (2.12)-(2.16), with initial condition $\xi_{i}(\theta)=\xi_{i, 0}(\theta)$ for $\theta \in[-\delta, 0) \quad(i=1, \ldots, N)$, $(z(0), w(0))=\left(z_{0}, w_{0}\right), \quad x(\theta)=x_{0}(\theta)$ for $\theta \in[-r, 0]$, $u(\theta)=u_{0}(\theta)$ for $\theta \in[-r-\tau, 0)$ corresponding to input $e \in L_{\text {loc }}^{\infty}\left(\Re_{+} ; \mathfrak{R}^{k}\right)$.

We prove next that the solution exists for all $t \geq 0$. In order to prove that the solution exists for all $t \geq 0$, it suffices to show that the solution exists and is bounded for all $t \in$ $\left[0, \tau_{1}\right)$. Indeed, if the solution exists and is bounded for all $t \in\left[0, \tau_{1}\right)$ then $x\left(\tau_{1}\right)$ and $z\left(\tau_{1}\right)$ can be uniquely defined and consequently $w\left(\tau_{1}\right)$ can be uniquely defined (by means of (2.14)). Therefore, all arguments can be repeated to the interval $\left[\tau_{1}, \tau_{2}\right)$ and in the same way we obtain existence of solution for all intervals $\left[\tau_{i}, \tau_{i+1}\right)(i=0,1,2, \ldots)$.

Standard results in ordinary differential equations guarantee that the system

$$
\begin{aligned}
\dot{z}(t) & =f(z(t), u(t-r-\tau))+\hat{k}(z(t), w(t), u(t-r-\tau)) \\
\dot{w}(t) & =\nabla h(z(t)) f(z(t), u(t-r-\tau))
\end{aligned}
$$

has a local solution defined on $\left[0, t_{1}\right)$ for some $t_{1} \in$ $\left(0, \min \left(\tau_{1}, r+\tau\right)\right]$. By virtue of (3.1) and Lemma 2.1, it follows that the solution of (3.2) satisfies the following estimate:

$$
V(z(t)) \leq \max \left(V\left(z_{0}\right), b\right)
$$

for all $t \geq 0$ for which the solution of (3.2) exists. Define the non-decreasing function:

$$
\begin{gathered}
\Omega(s):=\max \left\{|\nabla h(z) f(z, u)|:(z, u) \in \Re^{n} \times U,\right. \\
V(z) \leq s\}, \text { for all } s \geq \min \left(V(z): z \in \Re^{n}\right)
\end{gathered}
$$

which is well-defined by virtue of the facts that $U \subseteq \Re^{m}$ is compact and $V \in C^{2}\left(\Re^{n} ; \Re_{+}\right)$is a radially unbounded function. It follows from definition (3.4) and inequality (3.3) that the solution of (3.2) satisfies the following estimate for all $t \in\left[0, t_{1}\right)$ :

$$
|w(t)| \leq\left|w_{0}\right|+T_{s} \Omega\left(\max \left(V\left(z_{0}\right), b\right)\right) .
$$

A standard contradiction argument shows that the solution of (3.2) exists and satisfies (3.3), (3.5) for all $t \in$ $\left[0, \min \left(\tau_{1}, r+\tau\right)\right)$.

Next, consider the solution of the system (2.15) and (2.16). System (2.15) and (2.16) is a system described by IDEs with input $z(t)$. The existence of $t_{1} \in\left(0, \min \left(\tau_{1}, r+\tau\right)\right]$ for which the solution of systems (2.15) and (2.16) is uniquely defined on $\left[0, t_{1}\right)$ is a direct consequence of Theorem 2.1 in Karafyllis and Krstic (2013b) in conjunction with the fact that assumptions $\mathrm{H} 1$ and $\mathrm{H} 2$ in Karafyllis and Krstic (2013b) hold for systems (2.15) and (2.16). Using the fact that the inequality $q\left(\frac{|\xi|}{\psi(z)}\right) \xi \leq K \psi(z)$ holds for all $(\xi, z) \in \mathfrak{R}^{n} \times \mathfrak{R}^{n}$ in conjunction with definition $\tilde{p}(s):=$ $\max \left\{|f(z, u)|:(z, u) \in \mathfrak{R}^{n} \times U,|z| \leq s\right\}$, we obtain the estimate

$$
\left|\xi_{j}(t)\right| \leq K \psi(z(t))+\delta \tilde{p}(K \psi(z(t))), j=1, \ldots, N
$$

for $t \in\left[0, t_{1}\right)$ a.e. The fact that system (2.15) and (2.16) satisfies the boundedness-implies-continuation property (a consequence of Theorem 2.1 in Karafyllis and Krstic (2013b)) in conjunction with estimates (3.3) and (3.6) shows that the solution of (2.15) and (2.16) exists and satisfies (3.3) and (3.6) for $t \in\left[0, \min \left(\tau_{1}, r+\tau\right)\right)$ a.e.

Finally, the solution of (1.1) exists locally and by virtue of (2.1) and Lemma 2.1 satisfies the estimate:

$$
V(x(t)) \leq \max \left(V\left(x_{0}(0)\right), R\right)
$$

for all $t \geq 0$ for which the solution of (1.1) exists. A standard contradiction argument in conjunction with the fact that $V \in C^{2}\left(\Re^{n} ; \Re_{+}\right)$is a radially unbounded function guarantees that the solution of (1.1) exists and satisfies (3.7) for all $t \in\left[0, \min \left(\tau_{1}, r+\tau\right)\right)$.

If $r+\tau<\tau_{1}$ then all arguments may be repeated for the interval $t \in\left[r+\tau, \min \left(\tau_{1}, 2 r+2 \tau\right)\right)$ and continuing in this way we show that the solution of (1.1) and (2.12)-(2.16) exists for all $t \in\left[0, \tau_{1}\right)$. 
Lemma 2.1 in conjunction with (2.1) and (3.1) implies there exists $T \in C^{0}\left(\Re^{n} ; \Re_{+}\right)$such that (3.3), (3.7) hold for all $t \geq 0$ and

$V(x(t)) \leq R$ for all $t \geq T\left(x_{0}(0)\right)$ and $V(z(t)) \leq b$

for all $t \geq T\left(z_{0}\right)$.

Indeed the above conclusions for $V(x(t))$ are direct consequences of Lemma 2.1. The above conclusions for $V(z(t))$ are consequences of Lemma 2.1 applied to system (2.12) with $(w, u)$ as inputs. Inequalities (3.6) and (3.8) show that

$$
x(t) \in S_{1}, z(t) \in S_{2}, \xi_{j}(t) \in S_{3}(j=1, \ldots, N),
$$$$
\text { for } t \geq \max \left(T\left(x_{0}(0)\right), T\left(z_{0}\right)\right) \text { a.e., }
$$

where $S_{1}:=\left\{x \in \Re^{n}: V(x) \leq R\right\}, S_{2}:=\left\{x \in \Re^{n}: V(x)\right.$ $\leq b\}, S_{3}:=\left\{x \in \Re^{n}:|x| \leq K \psi(z)+\delta p(K \psi(z)), z \in S_{2}\right\}$.

Equation (1.1) implies that $x(t-r+j \delta)=$ $x(t-r+(j-1) \delta)+\int_{0}^{\delta} f(x(t-r+s+(j-1) \delta), u(t$ $+(j-1) \delta-\tau-r+s)) d s$ for $j=1, \ldots, N$ and $t \geq r$. Using the previous equation in conjunction with (2.1), implication (2.10) and Lemma 2.1 (which imply that $q\left(\frac{|x(t-r+s+(j-1) \delta)|}{\psi(x(t-r))}\right)=1$ for all $s \geq 0, t \geq r$ and $j=1, \ldots, N)$, we get from (2.15):

$$
\begin{aligned}
& \xi_{j}(t)-x(t-r+j \delta)=q\left(\frac{\left|\xi_{j-1}(t)\right|}{\psi(z(t))}\right) \xi_{j-1}(t) \\
& -q\left(\frac{|x(t-r+(j-1) \delta)|}{\psi(x(t-r))}\right) x(t-r+(j-1) \delta) \\
& +\int_{0}^{\delta} f\left(q\left(\frac{\left|\xi_{j}(t+s-\delta)\right|}{\psi(z(t))}\right) \xi_{j}(t+s-\delta),\right. \\
& u(t+(j-1) \delta-\tau-r+s)) d s \\
& -\int_{0}^{\delta} f\left(q\left(\frac{|x(t-r+s+(j-1) \delta)|}{\psi(x(t-r))}\right) x(t-r\right. \\
& +s+(j-1) \delta), u(t+(j-1) \delta-\tau-r+s)) d s
\end{aligned}
$$

for all $j=1, \ldots, N$ and $t \geq r$. Equation (3.10) in conjunction with (3.9) the fact that the inequality $q\left(\frac{|\xi|}{\psi(z)}\right) \xi \leq$ $K \psi(z)$ holds for all $(\xi, z) \in \Re^{n} \times \Re^{n}$ and the definitions

$$
\begin{aligned}
M_{1}^{q}:=\sup & \left\{\frac{\left|q\left(\frac{|\xi|}{\psi(z)}\right) \xi-q\left(\frac{|x|}{\psi(z)}\right) x\right|}{|x-\xi|}: x \in S_{1}, \xi \in S_{3},\right. \\
z & \left.\in S_{2}, x \neq \xi\right\},
\end{aligned}
$$

$$
\begin{aligned}
M_{2}^{q}:=\sup \left\{\frac{\left|q\left(\frac{|x|}{\psi(z)}\right) x-q\left(\frac{|x|}{\psi(w)}\right) x\right|}{|z-w|}: x, w \in S_{1},\right. \\
\left.\quad z \in S_{2}, z \neq w\right\}, \\
M_{1}^{f}:=\sup \left\{\frac{|f(x, u)-f(\xi, u)|}{|x-\xi|}: x \in S_{1}, \quad \xi \in S_{4},\right. \\
u \in U, x \neq \xi\}, \\
S_{4}:=\left\{x \in \Re^{n}:|x| \leq K \psi(z), z \in S_{2}\right\},
\end{aligned}
$$

implies that:

$$
\begin{aligned}
& \left|\xi_{j}(t)-x(t-r+j \delta)\right| \leq M_{1}^{q} \mid \xi_{j-1}(t)-x(t-r \\
& \quad+(j-1) \delta)\left|+\left(1+\delta M_{1}^{f}\right) M_{2}^{q}\right| z(t)-x(t-r) \mid \\
& \quad+\delta M_{1}^{f} M_{1}^{q} \sup _{-\delta \leq \theta \leq 0}\left(\left|\xi_{j}(t+\theta)-x(t+\theta-r+j \delta)\right|\right)
\end{aligned}
$$

for all $j=1, \ldots, N$ and $t \geq \max \left(T\left(x_{0}(0)\right), T\left(z_{0}\right)\right)+$ $\max (r, \delta)$. Using (3.11) in conjunction with (2.11), we obtain

$$
\begin{aligned}
& \sup _{t_{0} \leq t \leq T}\left(\left|\xi_{j}(t)-x(t-r+j \delta)\right| e^{\sigma t}\right) \leq \frac{M_{1}^{q}}{1-\delta M_{1}^{q} M_{1}^{f} e^{\sigma \delta}} \\
& \quad \times \sup _{t_{0} \leq t \leq T}\left(\left|\xi_{j-1}(t)-x(t-r+(j-1) \delta)\right| e^{\sigma t}\right) \\
& \quad+\frac{\left(1+\delta M_{1}^{f}\right) M_{2}^{q}}{1-\delta M_{1}^{q} M_{1}^{f} e^{\sigma \delta}} \sup _{t_{0} \leq t \leq T}\left(|z(t)-x(t-r)| e^{\sigma t}\right) \\
& +\sup _{t_{0}-\delta \leq t \leq t_{0}}\left(\left|\xi_{j}(t)-x(t-r+j \delta)\right| e^{\sigma t}\right)
\end{aligned}
$$

for all $j=1, \ldots, N \quad$ and $\quad T \geq t_{0}:=$ $\max \left(T\left(x_{0}(0)\right), T\left(z_{0}\right)\right)+\max (r, \delta)$. Using (3.12), we conclude that the following estimate holds:

$$
\begin{aligned}
& \sup _{t_{0} \leq s \leq t}\left(\left|\xi_{j}(s)-x(s+\tau)\right| e^{\sigma s}\right) \\
& \quad \leq\left(\frac{\left(1+\delta M_{1}^{f}\right) M_{2}^{q}}{1-\delta M_{1}^{q} M_{1}^{f} e^{\sigma \delta}}\left(1+\ldots+\lambda^{j-1}\right)+\lambda^{j}\right) \\
& \quad \times \sup _{t_{0} \leq s \leq t}\left(|z(s)-x(s-r)| e^{\sigma s}\right) \\
& \quad+\sum_{l=1}^{j} \lambda^{j-l} \sup _{t_{0}-\delta \leq s \leq t_{0}}\left(\left|\xi_{l}(s)-x(s-r+l \delta)\right| e^{\sigma s}\right)
\end{aligned}
$$


for all $j=1, \ldots, N \quad$ and $\quad t \geq t_{0}:=$ $\max \left(T\left(x_{0}(0)\right), T\left(z_{0}\right)\right)+\max (r, \delta)$ with $\lambda:=\frac{\bar{M}_{1}^{q}}{1-\delta M_{1}^{q} M_{1}^{f} e^{\sigma \delta}}$.

Next, consider the evolution of the mapping $t \rightarrow$ $P(x(t))$. Inequalities (2.2) and (3.9) imply that the following differential inequality holds for $t \geq \tau+$ $\max \left(T\left(x_{0}(0)\right), T\left(z_{0}\right)\right)$ a.e.:

$$
\begin{aligned}
& \frac{d}{d t}(P(x(t))) \leq-2 \mu|x(t)|^{2}+\sqrt{n} M_{2}^{f} \tilde{P}|x(t)| \\
& \quad \times\left|x(t)-\xi_{N}(t-\tau)\right|
\end{aligned}
$$

where $\quad M_{2}^{f}:=\sup \left\{\frac{|f(x, k(x))-f(x, k(\xi))|}{|x-\xi|}: x \in S_{1}, \quad \xi \in S_{3}\right.$, $x \neq \xi\}, \quad \tilde{P}:=\max \left\{\left|\nabla^{2} P(x)\right|: x \in \operatorname{co}\left(S_{1}\right)\right\}$ and $\operatorname{co}\left(S_{1}\right)$ denotes the convex hull of $S_{1}$. Completing the squares, integrating and noticing that there exists a constant $K_{1}>0$ with $K_{1}|x|^{2} \leq x^{\prime} P x \leq \frac{\sqrt{n}}{2} \tilde{P}|x|^{2} \quad$ for all $x \in S_{1}$, we obtain the following estimate for $t \geq \tau+t_{0}$, $t_{0}:=\max \left(T\left(x_{0}(0)\right), T\left(z_{0}\right)\right)+\max (r, \delta)$ :

$$
\begin{aligned}
&|x(t)| \leq e^{-\frac{2 \mu}{\sqrt{n} \tilde{P}}\left(t-t_{0}-\tau\right)} \sqrt{\frac{\sqrt{n} \tilde{P}}{2 K_{1}}}\left|x\left(t_{0}+\tau\right)\right| \\
& \quad+\sqrt{\frac{n \sqrt{n} \tilde{P}}{K_{1}}} \frac{M_{2}^{f} \tilde{P}}{2 \mu} \sup _{t_{0}+\tau \leq s \leq t} \\
& \times\left(e^{-\frac{\mu}{\sqrt{n} \tilde{P}}(t-s)}\left|x(s)-\xi_{N}(s-\tau)\right|\right) .
\end{aligned}
$$

Since $\sigma \leq \frac{\mu}{\sqrt{n} \tilde{P}}$ (see (2.11)), we obtain from (3.15) and (3.13) for $t \geq \tau+t_{0}$ :

$$
\begin{aligned}
& \sup _{t_{0}+\tau \leq s \leq t}\left(|x(s)| e^{\sigma s}\right) \leq \sqrt{\frac{\sqrt{n} \tilde{P}}{2 K_{1}}}\left|x\left(t_{0}+\tau\right)\right| e^{\sigma\left(\tau+t_{0}\right)} \\
& +\sqrt{\frac{n \sqrt{n} \tilde{P}}{K_{1}}} \frac{M_{2}^{f} \tilde{P}}{2 \mu}\left(\frac{\left(1+\delta M_{1}^{f}\right) M_{2}^{q}}{1-\delta M_{1}^{q} M_{1}^{f} e^{\sigma \delta}}(1+\ldots\right. \\
& \left.\left.+\lambda^{N-1}\right)+\lambda^{N}\right) \sup _{t_{0} \leq s \leq t-\tau}\left(|z(s)-x(s-r)| e^{\sigma s}\right) \\
& +\sqrt{\frac{n \sqrt{n} \tilde{P}}{K_{1}}} \frac{M_{2}^{f} \tilde{P}}{2 \mu} \sum_{l=1}^{N} \lambda^{N-l} \\
& \times \sup _{t_{0}-\delta \leq s \leq t_{0}}\left(\left|\xi_{l}(s)-x(s-r+l \delta)\right| e^{\sigma s}\right)
\end{aligned}
$$

Next, we establish the following inequality:

$$
\begin{aligned}
& (z-x)^{\prime} Q(f(z, u)+\hat{k}(z, h(x), u)-f(x, u)) \\
& \quad \leq-c \omega|z-x|^{2}, \text { for all }(x, z, u) \in S_{1} \times S_{2} \times U .
\end{aligned}
$$

Notice that inequality (2.3) and definitions (2.7)-(2.9) imply that (3.17) holds for the case $V(z) \leq a$. Therefore, we focus on the case $a<V(z) \leq b$. Definition (2.8) gives

$$
\begin{gathered}
(z-x)^{\prime} Q(f(z, u)+\hat{k}(z, h(x), u)-f(x, u)) \\
\leq(z-x)^{\prime} Q(f(z, u)+L(h(z)-h(x))-f(x, u)) \\
\quad-\frac{\varphi(z, h(x), u)}{|\nabla V(z)|^{2}} \nabla V(z) Q(z-x) .
\end{gathered}
$$

Inequalities (2.3), (3.18) and the fact that $\varphi(z, h(x), u) \geq 0$ implies that (3.17) holds if $\nabla V(z) Q(z-x) \geq 0$. Moreover, inequalities (2.3), (3.18) show that (3.17) holds if $\varphi(z, h(x), u)=0$. It remains to consider the case $\nabla V(z) Q(z-x)<0$ and $\varphi(z, h(x), u)>0$. In this case, definition (2.9) implies $\varphi(z, h(x), u)=\nabla V(z) f(z, u)+$ $W(z)+p(V(z)) \nabla V(z) L(h(z)-h(x))>0$. Then, inequality (2.6) gives

$$
\begin{aligned}
& \varphi(z, h(x), u)) \\
& =\nabla V(z) f(z, u)+p(V(z)) \nabla V(z) L(h(z)-h(x))+W(z) \\
& \leq+(1-p(V(z))) \nabla V(z) f(z, u)+(1-p(V(z))) W(z) \\
& \quad+(1-c)|\nabla V(z)|^{2} p(V(z)) \\
& \quad \times \frac{(z-x)^{\prime} Q(f(z, u)+L(h(z)-h(x))-f(x, u))}{\nabla V(\xi) Q(z-x)} .
\end{aligned}
$$

Using (3.19), (2.1) and the fact that $0 \leq p(V(z)) \leq 1$, we obtain

$$
\begin{aligned}
- & \frac{\varphi(z, h(x), u)) \nabla V(z) Q(z-x)}{|\nabla V(z)|^{2}} \leq \\
& -\frac{1-p(V(z))}{|\nabla V(z)|^{2}} \nabla V(z) Q(z-x)(\nabla V(z) f(z, u)+W(z)) \\
& -(1-c) p(V(z))(z-x)^{\prime} Q(f(z, u) \\
& +L(h(z)-h(x))-f(x, u)) \leq-(1-c)(z-x)^{\prime} \\
& \times Q(f(z, u)+L(h(z)-h(x))-f(x, u)) .
\end{aligned}
$$

Combining (2.3), (3.18) and the above inequality, we conclude that (3.17) holds.

Next, consider the evolution of the mapping $t \rightarrow$ $(z(t)-x(t-r))^{\prime} Q(z(t)-x(t-r))$. Inequality (3.17) and (3.9) imply that the following differential inequality holds for $t \geq r+\max \left(T\left(x_{0}(0)\right), T\left(z_{0}\right)\right)$ a.e.:

$$
\begin{aligned}
& \frac{d}{d t}\left((z(t)-x(t-r))^{\prime} Q(z(t)-x(t-r))\right) \\
& \leq-2 c \omega|z(t)-x(t-r)|^{2}+2 G_{2}|Q| \\
& \quad \times|z(t)-x(t-r)||w(t)-h(x(t-r))|,
\end{aligned}
$$

where $\quad G_{2}:=\sup \left\{\frac{|\hat{k}(z, y, u)-\hat{k}(z, w, u)|}{|y-w|}: y, w \in \Re^{k}, z \in S_{2}\right.$, $u \in U, y \neq w\}$. Since $Q \in \Re^{n \times n}$ is a positive definite matrix there exists a constant $0<K_{2} \leq|Q|$ with 
$K_{2}|x|^{2} \leq x^{\prime} Q x$ for all $x \in \Re^{n}$. Completing the squares and integrating we obtain the following estimate for $t \geq t_{0}$, $t_{0}:=\max \left(T\left(x_{0}(0)\right), T\left(z_{0}\right)\right)+\max (r, \delta)$ :

$$
\begin{aligned}
& |z(t)-x(t-r)| \leq e^{-\frac{c \omega}{2|Q|}\left(t-t_{0}\right)} \sqrt{\frac{|Q|}{K_{2}}}\left|z\left(t_{0}\right)-x\left(t_{0}-r\right)\right| \\
& +\sqrt{\frac{2|Q|}{K_{2}}} \frac{G_{2}|Q|}{c \omega} \sup _{t_{0} \leq s \leq t}\left(e^{-\frac{c \omega}{4|Q|}(t-s)}|w(s)-h(x(s-r))|\right) .
\end{aligned}
$$

Since $\sigma \leq \frac{c \omega}{4|Q|}($ see (2.11)), we obtain from (3.21) for $t \geq$ $t_{0}$ :

$$
\begin{gathered}
\sup _{t_{0} \leq s \leq t}\left(e^{\sigma s}|z(s)-x(s-r)|\right) \leq \sqrt{\frac{|Q|}{K_{2}}} e^{\sigma t_{0}}\left|z\left(t_{0}\right)-x\left(t_{0}-r\right)\right| \\
+\sqrt{\frac{2|Q|}{K_{2}}} \frac{G_{2}|Q|}{c \omega} \sup _{t_{0} \leq s \leq t}\left(e^{\sigma s}|w(s)-h(x(s-r))|\right) .
\end{gathered}
$$

Finally, notice that since $\sup _{i \geq 0}\left(\tau_{i+1}-\tau_{i}\right) \leq T_{s}$ the following estimate holds for every $t \in\left[\tau_{i}, \tau_{i+1}\right)$ with $\tau_{i} \geq t_{0}$ :

$$
\begin{aligned}
& |w(t)-h(x(t-r))| \leq \sup _{0 \leq s \leq t}|e(s)|+T_{s} G_{1} \\
& \quad \times \sup _{\tau_{i} \leq s \leq t}|z(s)-x(s-r)|,
\end{aligned}
$$

where $G_{1}:=\sup \left\{\frac{|\nabla h(x) f(x, u)-\nabla h(z) f(z, u)|}{|x-z|}: x \in S_{1}, \quad z \in S_{2}\right.$, $u \in U, x \neq z\}$. Note that from the inequalities $t \leq \tau_{i}+T_{s}$, $\tau_{i} \leq t_{0}+T_{s}$ and (3.23) we obtain for all $t \geq t_{0}+T_{s}$ :

$$
\begin{aligned}
& \sup _{t_{0}+T_{s} \leq s \leq t}\left(e^{\sigma s}|w(s)-h(x(s-r))|\right) \leq e^{\sigma t} \sup _{0 \leq s \leq t}|e(s)| \\
& \quad+T_{s} G_{1} e^{\sigma T_{s}} \sup _{t_{0} \leq s \leq t}\left(e^{\sigma s}|z(s)-x(s-r)|\right)
\end{aligned}
$$

Combining (3.22) and (3.24), we get for all $t \geq t_{0}+T_{s}$ :

$$
\begin{aligned}
& \sup _{t_{0} \leq s \leq t}\left(e^{\sigma s}|z(s)-x(s-r)|\right) \leq \sqrt{\frac{|Q|}{K_{2}}} e^{\sigma t_{0}} \\
& \quad \times\left|z\left(t_{0}\right)-x\left(t_{0}-r\right)\right|+\sqrt{\frac{2|Q|}{K_{2}}} \frac{G_{2}|Q|}{c \omega} e^{\sigma t} \sup _{0 \leq s \leq t}(|e(s)|) \\
& \quad+T_{s} G_{1} e^{\sigma T_{s}} \sqrt{\frac{2|Q|}{K_{2}}} \frac{G_{2}|Q|}{c \omega} \sup _{t_{0} \leq s \leq t}\left(e^{\sigma s}|z(s)-x(s-r)|\right) \\
& \quad+\sqrt{\frac{2|Q|}{K_{2}}} \frac{G_{2}|Q|}{c \omega} \sup _{t_{0} \leq s \leq t_{0}+T_{s}}\left(e^{\sigma s}|w(s)-h(x(s-r))|\right) .
\end{aligned}
$$

It follows from (2.11) and (3.25) that the following estimate holds for all $t \geq t_{0}+T_{s}$ :

$$
\begin{aligned}
\sup _{t_{0} \leq s \leq t}\left(e^{\sigma s}|z(s)-x(s-r)|\right) \\
\quad \leq \frac{c \omega \sqrt{|Q|}}{c \omega \sqrt{K_{2}}-T_{s} G_{1} G_{2}|Q| e^{\sigma T_{s}} \sqrt{2|Q|}} e^{\sigma t_{0}} \\
\quad \times\left|z\left(t_{0}\right)-x\left(t_{0}-r\right)\right| \\
\quad+\frac{G_{2}|Q| \sqrt{2|Q|}}{c \omega \sqrt{K_{2}}-T_{s} G_{1} G_{2}|Q| e^{\sigma T_{s}} \sqrt{2|Q|}} \\
\quad \times\left(e^{\sigma t} \sup _{0 \leq s \leq t}(|e(s)|)+\sup _{t_{0} \leq s \leq t_{0}+T_{s}}\left(e^{\sigma s} \mid w(s)\right.\right. \\
-h(x(s-r)) \mid)) .
\end{aligned}
$$

Combining (3.16) and (3.26), we obtain the following inequality for all $t \geq t_{0}+T_{s}$ :

$$
\begin{aligned}
& |x(t)| e^{\sigma t} \leq A_{1}\left|x\left(t_{0}+\tau\right)\right| e^{\sigma\left(\tau+t_{0}\right)} \\
& \quad+A_{2} \sup _{t_{0} \leq s \leq t_{0}+T_{s}}\left(|w(s)-h(x(s-r))| e^{\sigma s}\right) \\
& \quad+A_{3} e^{\sigma t_{0}}\left|z\left(t_{0}\right)-x\left(t_{0}-r\right)\right|+A_{4} e^{\sigma t} \sup _{0 \leq s \leq t}(|e(s)|) \\
& \quad+A_{5} \sum_{l=1}^{N} \sup _{t_{0}-\delta \leq s \leq t_{0}}\left(\left|\xi_{l}(s)-x(s-r+l \delta)\right| e^{\sigma s}\right)
\end{aligned}
$$

for appropriate constants $A_{i}>0(i=1, \ldots, 5)$. Combining (3.13), (3.24), (3.26), (3.27), using (2.16) and the fact that $k: \Re^{n} \rightarrow U$ is globally Lipschitz with $k(0)=0$ and defining

$$
T_{0}:=\max \left(T\left(x_{0}(0)\right), T\left(z_{0}\right)\right)+r+2 \tau+T_{s},
$$

we obtain the following estimate for all $t \geq 0$ :

$$
\begin{aligned}
& \sup _{-r \leq s \leq 0}(|x(t+s)|)+|w(t)|+|z(t)|+\sum_{j=1}^{N} \sup _{-\delta \leq s<0} \\
& \quad \times\left(\left|\xi_{j}(t+s)\right|\right)+\sup _{-r-\tau \leq s<0}(|u(t+s)|) \\
& \quad \leq A e^{-\sigma\left(t-T_{0}\right)}\left(\sup _{-r \leq s \leq T_{0}}(|x(s)|)+\sup _{0 \leq s \leq T_{0}}(|z(s)|)\right. \\
& +\sup _{0 \leq s \leq T_{0}}(|w(s)|)+\sum_{j=1}^{N} \sup _{-\delta \leq s<T_{0}}\left(\left|\xi_{j}(s)\right|\right) \\
& \left.+\sup _{-r-\tau \leq s<T_{0}}(|u(s)|)\right)+\gamma \sup _{0 \leq s \leq t}(|e(s)|)
\end{aligned}
$$

for appropriate constants $A, \gamma>0$.

Estimates (3.3), (3.6), (3.7) and the fact that $k: \Re^{n} \rightarrow$ $U$ is globally Lipschitz with $k(0)=0$ guarantee that there exists a non-decreasing, smooth function $\Delta: \Re_{+} \rightarrow \Re_{+}$ 
such that

$$
\begin{aligned}
& \sup _{-r \leq s \leq 0}(|x(t+s)|)+|z(t)|+\sum_{j=1}^{N} \sup _{-\delta \leq s<0}\left(\left|\xi_{j}(t+s)\right|\right) \\
& +\sup _{-r-\tau \leq s<0}(|u(t+s)|) \leq \Delta\left(\sup _{-r \leq s \leq 0}(|x(s)|)+|z(0)|\right. \\
& \left.+\sum_{j=1}^{N} \sup _{-\delta \leq s<0}\left(\left|\xi_{j}(s)\right|\right)+\sup _{-r-\tau \leq s<0}(|u(s)|)\right)
\end{aligned}
$$

for all $t \geq 0$. Definitions (2.7)-(2.9) in conjunction with the fact that $q(s) \leq K$ for all $s \geq 0$, guarantee the existence of a smooth, non-decreasing function $G: \Re_{+} \rightarrow \Re_{+}$such that the following inequality holds for every $R \geq 0$ :

$$
\begin{aligned}
& \left|f\left(q\left(\frac{|\xi|}{\psi(z)}\right) \xi, u_{1}\right)\right|+\left|f\left(x, u_{2}\right)\right|+\left|\nabla h(z) f\left(z, u_{3}\right)\right| \\
& \quad+\left|f\left(z, u_{3}\right)+\hat{k}\left(z, w, u_{3}\right)\right|+|h(\zeta)|, \\
& \leq G(R)\left(|\xi|+|x|+|z|+|w|+\left|u_{1}\right|+\left|u_{2}\right|+\left|u_{3}\right|+|\zeta|\right) \\
& \quad \text { for all }(\xi, \zeta, x, z) \in\left(\Re^{n}\right)^{4}, w \in \Re^{k},\left(u_{1}, u_{2}, u_{3}\right) \in U^{3} \\
& \quad \text { with }|\xi|+\max (|x|,|\zeta|)+|z|+\max _{i=1,2,3}\left|u_{i}\right| \leq R .
\end{aligned}
$$

In order to finish the proof, we notice that is suffices to prove that there exist smooth functions $p_{j}: \Re_{+} \times \Re_{+} \rightarrow$ $\Re_{+}$for which $p_{j}(R, \cdot)$ and $p_{j}(\cdot, t)$ are non-decreasing for every fixed $(R, t) \in \Re_{+} \times \Re_{+}(j=1,2,3,4)$ such that the following estimates

$$
\begin{aligned}
|x(t)| \leq & p_{1}(R, t)\left(\sup _{-r \leq s \leq 0}(|x(s)|)+|z(0)|+|w(0)|\right. \\
& +\sum_{j=1}^{N} \sup _{-\delta \leq s<0}\left(\left|\xi_{j}(s)\right|\right)+\sup _{-r-\tau \leq s<0}(|u(s)|) \\
& \left.+\sup _{0 \leq s \leq t}(|e(s)|)\right) \\
|z(t)| \leq & p_{2}(R, t)\left(\sup _{-r \leq s \leq 0}(|x(s)|)+|z(0)|+|w(0)|\right. \\
& +\sum_{j=1}^{N} \sup _{-\delta \leq s<0}\left(\left|\xi_{j}(s)\right|\right)+\sup _{-r-\tau \leq s<0}(|u(s)|) \\
& \left.+\sup _{0 \leq s \leq t}(|e(s)|)\right) \\
|w(t)| \leq & p_{3}(R, t)\left(\sup _{-r \leq s \leq 0}(|x(s)|)+|z(0)|+|w(0)|\right. \\
& +\sum_{j=1}^{N} \sup _{-\delta \leq s<0}\left(\left|\xi_{j}(s)\right|\right)+\sup _{-r-\tau \leq s<0}(|u(s)|) \\
& \left.+\sup _{0 \leq s \leq t}(|e(s)|)\right)
\end{aligned}
$$

$$
\begin{aligned}
& |u(t)|+\sum_{j=1}^{N}\left|\xi_{j}(t)\right| \leq p_{4}(R, t)\left(\sup _{-r \leq s \leq 0}(|x(s)|)+|z(0)|\right. \\
& \quad+|w(0)|+\sum_{j=1}^{N} \sup _{-\delta \leq s<0}\left(\left|\xi_{j}(s)\right|\right)+\sup _{-r-\tau \leq s<0}(|u(s)|) \\
& \left.\quad+\sup _{0 \leq s \leq t}(|e(s)|)\right)
\end{aligned}
$$

hold for all $t \geq 0$ with $R:=\sup _{-r \leq s \leq 0}(|x(s)|)+|z(0)|+$ $\sum_{j=1}^{N} \sup _{-\delta \leq s<0}\left(\left|\xi_{j}(s)\right|\right)+\sup _{-r-\tau \leq s<0}(|u(s)|)$. Indeed, if estimates (3.32)-(3.35) hold then by virtue of (3.28) and (3.29) we conclude that (2.17) holds with $\Gamma:=\gamma$ and $C(s):=s A e^{\sigma \tilde{T}(s)} \max _{j=1,2,3,4}\left(p_{j}(s, \tilde{T}(s))\right)$ for all $s \geq 0$, where $\tilde{T}: \Re_{+} \rightarrow \Re_{+}$is a smooth, non-decreasing function that satisfies

$r+2 \tau+T_{s}+\max \{T(x):|x| \leq s\} \leq \tilde{T}(s)$, for all $s \geq 0$.

For convenience we use $G$ in order to denote $G(\Delta(R)) \quad$ with $\quad R:=\sup _{-r \leq s \leq 0}(|x(s)|)+|z(0)|+\sum_{j=1}^{N}$ $\sup _{-\delta \leq s<0}\left(\left|\xi_{j}(s)\right|\right)+\sup _{-r-\tau \leq s<0}(|u(s)|)$. Using (2.12), (3.30) and (3.31), we get

$$
\begin{aligned}
|z(t)| \leq & |z(0)|+G \int_{0}^{t}|z(s)| d s+G \int_{0}^{t}|w(s)| d s \\
& +G \int_{0}^{t}|u(s-r-\tau)| d s
\end{aligned}
$$

for all $t \geq 0$. Applying the Gronwall-Belman lemma to (3.37), we obtain

$$
\begin{aligned}
|z(t)| \leq & e^{2 G t}\left(|z(0)|+G \int_{0}^{t}|w(s)| d s\right. \\
& \left.+G \int_{0}^{t}|u(s-r-\tau)| d s\right)
\end{aligned}
$$

for all $t \geq 0$. Using (2.13), (2.14), (3.30), and (3.31), we get

$$
\begin{aligned}
& |w(t)| \leq|w(0)|+G \sup _{-r \leq s \leq t-r}|x(s)|+G \sup _{0 \leq s \leq t}|e(s)| \\
& \quad+G \int_{\tau_{i}}^{t}|z(s)| d s+G \int_{\tau_{i}}^{t}|u(s-r-\tau)| d s
\end{aligned}
$$

for all $t \in\left[\tau_{i}, \tau_{i+1}\right)$. Since $t \leq \tau_{i}+T_{s}$, we obtain from (3.38) and (3.39) for all $t \in\left[\tau_{i}, \tau_{i+1}\right)$ :

$$
\begin{aligned}
& |w(t)| \leq|w(0)|+G \sup _{-r \leq s \leq t-r}|x(s)|+G \sup _{0 \leq s \leq t}|e(s)| \\
& \quad+G T_{s} e^{2 G t}|z(0)|+G^{2} T_{s} e^{2 G t} \int_{0}^{t}|w(s)| d s \\
& \quad+G\left(1+G T_{s} e^{2 G t}\right) \int_{0}^{t}|u(s-r-\tau)| d s
\end{aligned}
$$


Note that (3.40) holds for all $t \geq 0$. Applying the GronwallBelman lemma to (3.40) and noticing that the GronwallBelman lemma holds not only for continuous functions but also for piecewise continuous functions (and that the mapping $t \rightarrow|w(t)|$ is piecewise continuous on $\Re_{+}$), we get

$$
\begin{aligned}
& |w(t)| \leq(1+G)\left(1+G T_{s} e^{2 G t}\right) e^{G^{2} T_{s} e^{2 G t_{t}}} \\
& \quad \times\left(|w(0)|+\sup _{-r \leq s \leq t-r}|x(s)|+\sup _{0 \leq s \leq t}|e(s)|+|z(0)|\right. \\
& \left.\quad+\int_{0}^{t}|u(s-r-\tau)| d s\right)
\end{aligned}
$$

for all $t \geq 0$. Combining (3.38) with (3.41) we obtain

$$
\begin{aligned}
& |w(t)|+|z(t)| \leq a_{1}(R, t)\left(|w(0)|+\sup _{-r \leq s \leq t-r}|x(s)|\right. \\
& \left.+\sup _{0 \leq s \leq t}|e(s)|+|z(0)|+\int_{-r-\tau}^{t}|u(s)| d s\right)
\end{aligned}
$$

for all $t \geq 0$ with $a_{1}(R, t):=e^{3 G t}(1+G)\left(2+G T_{s} e^{2 G t}\right)$ $e^{G^{2} T_{s} e^{2 G t} t}$, where $G:=G(\Delta(R))$.

We next continue with $\left|\xi_{j}(t)\right|(j=1, \ldots, N)$. We notice that (2.15) shows the mappings $t \rightarrow \xi_{j}(t)$ are continuous for all $t \geq 0(j=1, \ldots, N)$. Using (2.15), (3.30), (3.11), in conjunction with the fact that $q(s) \leq K$ for all $s \geq 0$, we get

$$
\begin{aligned}
& \left|\xi_{j}(t)\right| \leq K\left|\xi_{j-1}(t)\right|+G \int_{t-\delta}^{t}\left|\xi_{j}(s)\right| d s \\
& \quad+G \int_{-r-\tau}^{t}|u(s)| d s
\end{aligned}
$$

for all $t \geq 0$ and $j=1, \ldots, N$. Inequality (3.43) implies that the following inequality

$$
\begin{aligned}
& \left|\xi_{j}(t)\right| \leq K\left|\xi_{j-1}(t)\right|+G \int_{0}^{t}\left|\xi_{j}(s)\right| d s \\
& \quad+G \int_{-\delta}^{0}\left|\xi_{j}(s)\right| d s+G \int_{-r-\tau}^{t}|u(s)| d s
\end{aligned}
$$

holds for all $t \geq 0$ and $j=1, \ldots, N$. Applying the Gronwall-Belman lemma to (3.44), we obtain

$$
\begin{aligned}
& \left|\xi_{j}(t)\right| \leq\left(1+G t e^{G t}\right)\left(K \sup _{0 \leq s \leq t}\left(\left|\xi_{j-1}(s)\right|\right)\right. \\
& \left.\quad+G \int_{-r-\tau}^{t}|u(s)| d s+G \delta \sup _{-\delta \leq s<0}\left(\left|\xi_{j}(s)\right|\right)\right)
\end{aligned}
$$

for all $t \geq 0$ and $j=1, \ldots, N$. Using induction, inequalities (3.42) and (3.45), the fact that $\xi_{0}(t)=z(t)$ and the fact that $K \geq 1$ we obtain

$$
\begin{aligned}
& \left|\xi_{j}(t)\right| \leq\left(1+G t e^{G t}\right)^{j} K^{j} a_{1}(R, t) \\
& \quad \times\left(|z(0)|+\sup _{-r \leq s \leq t-r}|x(s)|+\sup _{0 \leq s \leq t}|e(s)|+|w(0)|\right. \\
& +\left(1+\frac{2^{j-1} G}{K}\right) \int_{-r-\tau}^{t}|u(s)| d s \\
& \left.\quad+\frac{G \delta}{K} \sum_{l=1}^{j} \sup _{-\delta \leq s<0}\left(\left|\xi_{l}(s)\right|\right)\right)
\end{aligned}
$$

for all $t \geq 0$ and $j=1, \ldots, N$. Let $\Lambda \geq 0$ be a constant for which the inequality $|k(x)| \leq \Lambda|x|$ for all $x \in \Re^{n}$ (since $k: \Re^{n} \rightarrow U$ is globally Lipschitz with $k(0)=0$ the existence of $\Lambda \geq 0$ is guaranteed). Using (3.46) with $j=N$ and (2.16), we get

$$
\begin{aligned}
& |u(t)| \leq a_{2}(R, t)\left(|z(0)|+\sup _{-r \leq s \leq t-r}|x(s)|+\sup _{0 \leq s \leq t}|e(s)|\right. \\
& +|w(0)|+\left(1+\frac{2^{N-1} G}{K}\right) \int_{-r-\tau}^{t}|u(s)| d s \\
& \left.\quad+\frac{G \delta}{K} \sum_{l=1}^{N} \sup _{-\delta \leq s<0}\left(\left|\xi_{l}(s)\right|\right)\right)
\end{aligned}
$$

for all $t \geq 0$ with $a_{2}(R, t):=\Lambda\left(1+G t e^{G t}\right)^{N} K^{N} a_{1}(R, t)$, where $G:=G(\Delta(R))$. Applying the Gronwall-Belman lemma to (3.44) we obtain

$$
\begin{aligned}
& |u(t)| \leq a_{3}(R, t)\left(|z(0)|+\sup _{-r \leq s \leq t-r}|x(s)|+\sup _{0 \leq s \leq t}|e(s)|\right. \\
& +|w(0)|+\left(1+\frac{2^{N-1} G}{K}\right) \int_{-r-\tau}^{0}|u(s)| d s \\
& \left.+\frac{G \delta}{K} \sum_{l=1}^{N} \sup _{-\delta \leq s<0}\left(\left|\xi_{l}(s)\right|\right)\right)
\end{aligned}
$$

for all $t \geq 0$ with $a_{3}(R, t):=a_{2}(R, t) e^{a_{2}(R, t)\left(1+\frac{2^{N-1} G}{K}\right) t}$, where $G:=G(\Delta(R))$. Combining (3.42), (3.46) with (3.48) we obtain

$$
\begin{aligned}
& |w(t)|+|z(t)| \leq a_{4}(R, t)\left(|w(0)|+\sup _{-r \leq s \leq t-r}|x(s)|\right. \\
& +\sup _{0 \leq s \leq t}|e(s)|+|z(0)|+\int_{-r-\tau}^{0}|u(s)| d s \\
& \left.\quad+\sum_{l=1}^{N} \sup _{-\delta \leq s<0}\left(\left|\xi_{l}(s)\right|\right)\right)
\end{aligned}
$$


and

$$
\begin{aligned}
& |u(t)|+\sum_{j=1}^{N}\left|\xi_{j}(t)\right| \leq a_{5}(R, t)\left(|z(0)|+\sup _{-r \leq s \leq t-r}|x(s)|\right. \\
& +\sup _{0 \leq s \leq t}|e(s)|+|w(0)|+\int_{-r-\tau}^{0}|u(s)| d s \\
& \left.\quad+\sum_{l=1}^{N} \sup _{-\delta \leq s<0}\left(\left|\xi_{l}(s)\right|\right)\right)
\end{aligned}
$$

for all $t \geq 0$ with $a_{4}(R, t):=\left(2+\frac{\left(\delta+2^{N-1}\right) G}{K}\right)\left(1+t a_{3}(R, t)\right)$ $a_{1}(R, t), \quad a_{5}(R, t):=(N+\Lambda)\left(1+G t e^{G t}\right)^{N} K^{N} a_{1}(R, t)$ $\left(1+\frac{\left(\delta+2^{N-1}\right) G}{K}\right)^{2}\left(1+t a_{3}(R, t)\right)$, where $G:=G(\Delta(R))$.

Finally, using (1.1), (3.30) and (3.31), we get

$$
|x(t)| \leq|x(v)|+G \int_{v}^{t}|x(s)| d s+G \int_{v}^{t}|u(s-\tau)| d s
$$

for all $T \geq t \geq v \geq 0$. Combining (3.51) with (3.50), we get

$$
\begin{aligned}
& |x(t)| \leq G(t-v)\left(1+a_{5}(R, T)\right) \sup _{v \leq s \leq t}|x(s)|+(1+G \\
& \left.\quad+G(t-v) a_{5}(R, T)\right)\left(M+\sup _{-r \leq s \leq v}|x(s)|\right)
\end{aligned}
$$

for $\quad$ all $\quad T \geq t \geq v \geq 0, \quad$ where $\quad M:=|x(0)|+$ $|z(0)|+|w(0)|+\sup _{0 \leq s \leq T}|e(s)|+\int_{-r-\tau}^{0}|u(s)| d s+$ $\sum_{j=1}^{N} \sup _{-\delta \leq s<0}\left(\left|\xi_{j}(s)\right|\right)$. Let $\theta>0$ be a constant with $G \theta\left(1+a_{5}(R, T)\right) \leq 1 / 2$. It follows from (3.52) that the inequality

$$
|x(t)| \leq 2(2+G)\left(M+\sup _{-r \leq s \leq v}|x(s)|\right)
$$

holds for all $T \geq v \geq 0$ and $t \in[v, \min (v+\theta, T)]$. Applying (3.53) repeatedly, we get

$$
\begin{aligned}
& \sup _{-r \leq s \leq i \theta}|x(s)| \leq M\left(2(2+G)+\cdots+2^{i}(2+G)^{i}\right) \\
& +2^{i}(2+G)^{i} \sup _{-r \leq s \leq 0}|x(s)|
\end{aligned}
$$

for all $T \geq 0$ and all integers $i \geq 0$ with $i \theta \leq T$. Selecting $i \geq 0$ so that $i:=1+\left[2 G T\left(1+a_{5}(R, T)\right)\right]$ and $\theta=T / i$, we get (3.32) with $p_{1}(R, t):=$ $\left(1+2 G t\left(1+a_{5}(R, t)\right)\right)(2(2+G))^{\left(1+2 G t\left(1+a_{5}(R, t)\right)\right)}$.

Exploiting (3.32), (3.49) and (3.50), we get inequalities (3.33), (3.34) and (3.35) for appropriate smooth functions $p_{j}: \Re_{+} \times \Re_{+} \rightarrow \Re_{+}$for which $p_{j}(R, \cdot)$ and $p_{j}(\cdot, t)$ are non-decreasing for every fixed $(R, t) \in \Re_{+} \times \Re_{+}$ $(j=2,3,4)$. The proof is complete.
We next continue with the proof of Theorem 2.4.

Proof of Theorem 2.4: We first notice that if the solution of the closed-loop system (2.18) and (2.19) with (2.12)-(2.16) and (2.22)-(2.24) exists for all $t \geq 0$ then the following equalities hold:

$$
\begin{gathered}
\theta(t)=a_{1}(x(t+\tau)), \text { for all } t \geq \tau_{l} \\
\dot{x}(t)=f(x(t), u(t-\tau)), \text { for all } t \geq \tau_{l}+\tau \text { a.e. }
\end{gathered}
$$

where $\tau_{l}=\min \left\{\tau_{i}: i \geq 1, \quad \tau_{i} \geq r\right\}$. The above equalities are direct consequences of (2.21) and the fact that the equation $\nabla a_{1}(x) \tilde{f}(x, v)=g\left(a_{1}(x), v\right)$ holds for all $(x, v) \in$ $\Re^{n} \times \Re^{m}$. Therefore, by virtue of Theorem 2.2 , there exists a locally Lipschitz function $\tilde{C} \in K_{\infty}$ such that the solution of the closed-loop system (2.18) and (2.19) with (2.12)(2.16) and (2.22)-(2.24) satisfies the following estimate for all $t \geq \tau_{l}+\tau$ :

$$
\begin{aligned}
& \sup _{-r \leq s \leq 0}(|x(t+s)|)+|w(t)|+|z(t)| \\
& +\sum_{j=1}^{N} \sup _{-\delta \leq s<0}\left(\left|\xi_{j}(t+s)\right|\right)+\sup _{-r-\tau \leq s<0}(|u(t+s)|) \\
& \leq e^{-\sigma\left(t-\tau_{l}-\tau\right)} \tilde{C}\left(\sup _{-r \leq s \leq 0}\left(\left|x\left(\tau_{l}+\tau+s\right)\right|\right)+\left|z\left(\tau_{l}+\tau\right)\right|\right. \\
& +\left|w\left(\tau_{l}+\tau\right)\right|+\sum_{j=1}^{N} \sup _{\delta \leq s<0}\left(\left|\xi_{j}\left(\tau_{l}+\tau+s\right)\right|\right) \\
& \left.+\sup _{-r-\tau \leq s<0}\left(\left|u\left(\tau_{l}+\tau+s\right)\right|\right)\right) .
\end{aligned}
$$

Since $a_{1}: \Re^{n} \rightarrow \mathfrak{R}^{l}$ is a locally Lipschitz function with $a_{1}(0)=0$, there exists a non-decreasing function $c: \Re_{+} \rightarrow$ $\Re_{+}$such that $\left|a_{1}(x)\right| \leq|x| c(|x|)$ for all $x \in \Re^{n}$. The previous inequality in conjunction with (3.55) and (3.57) implies the existence of a locally Lipschitz function $\bar{C} \in K_{\infty}$ such that the solution of the closed-loop systems (2.18) and (2.19) with (2.12)-(2.16) and (2.22)-(2.24) satisfies the following estimate for all $t \geq \tau_{l}+\tau$ :

$$
\begin{aligned}
& \sup _{-r \leq s \leq 0}(|x(t+s)|)+|w(t)|+|\theta(t)|+|z(t)| \\
& +\sum_{j=1}^{N} \sup _{-\delta \leq s<0}\left(\left|\xi_{j}(t+s)\right|\right)+\sup _{-r-\tau \leq s<0}(|u(t+s)|) \\
& \leq e^{-\sigma\left(t-\tau_{l}-\tau\right)} \bar{C}\left(\sup _{-r \leq s \leq 0}\left(\left|x\left(\tau_{l}+\tau+s\right)\right|\right)+\left|z\left(\tau_{l}+\tau\right)\right|\right. \\
& +\left|w\left(\tau_{l}+\tau\right)\right|+\sum_{j=1}^{N} \sup _{\delta \leq s<0}\left(\left|\xi_{j}\left(\tau_{l}+\tau+s\right)\right|\right) \\
& \left.+\sup _{-r-\tau \leq s<0}\left(\left|u\left(\tau_{l}+\tau+s\right)\right|\right)\right) .
\end{aligned}
$$


Inequality (2.25) is a direct consequence of estimate (3.58). Therefore, in order to prove Theorem 2.4 it suffices to show that the solution of the closed-loop systems (2.18) and (2.19) with (2.12)-(2.16) and (2.22)-(2.24) exists for all $t \geq 0$.

The arguments for the proof of the existence of the solution the solution of the closed-loop systems (2.18) and (2.19) with (2.12)-(2.16) and (2.22)-(2.24) are exactly the same as those in the proof of the Theorem 2.2 except that

- we do not use (3.7) but instead we use the fact that (2.18) is forward complete in conjunction with the results in Angeli and Sontag (1999)), and

- we use the fact that system (2.20) is forward complete.

The proof is complete.

\section{Illustrative examples}

This section is devoted to the presentation of two nonlinear control systems that can be stabilised by the results of Theorems 2.2 and 2.4.

Example 4.1: This is an example of a two-dimensional nonlinear control system for which all assumptions $\mathrm{H} 1-\mathrm{H} 4$ hold. It follows from Theorem 2.2 that the system can be stabilised globally asymptotically and locally exponentially by means of the ISP-O-P-DFC control scheme. The system is described by the equations

$$
\begin{aligned}
\dot{x}_{1}(t) & =g x_{1}(t)-x_{1}^{3}(t)+x_{2}(t), \\
\dot{x}_{2}(t) & =-x_{2}^{3}(t)+u(t-\tau), \\
x(t) & =\left(x_{1}(t), x_{2}(t)\right) \in \mathfrak{R}^{2}, \\
u(t) & \in[-4 \sqrt{2}, 4 \sqrt{2}] \subset \Re,
\end{aligned}
$$

where $\tau \geq 0$ is the input delay and $g>0$ is a constant. The measured output is given by the equation:

$$
y\left(\tau_{i}\right)=x_{1}\left(\tau_{i}-r\right)+e\left(\tau_{i}\right),
$$

where $\left\{\tau_{i}\right\}_{i=0}^{\infty}$ is the sampling partition (a partition of $\Re_{+}$), $r \geq 0$ is the measurement delay and the input $e: \Re_{+} \rightarrow \mathfrak{R}$ is the measurement error. We show next that assumptions H1-H4 hold for system (4.1) provided that

$$
g \leq \frac{1}{167}
$$

We notice that systems (4.1) and (4.2) is a system of the forms (1.1) and (1.2) with $U=[-4 \sqrt{2}, 4 \sqrt{2}] \subset$ $\Re$ and $f(x, u):=\left[\begin{array}{c}g x_{1}-x_{1}^{3}+x_{2} \\ -x_{2}^{3}+u\end{array}\right]$. We start by showing that assumption $\mathrm{H} 1$ holds with $V(x):=\frac{1}{2} x_{1}^{2}+\frac{1}{2} x_{2}^{2}$,
$W(x):=\frac{1}{2} V(x)$ and $R:=4$. Indeed, using the inequalities $x_{1} x_{2} \leq \frac{1}{2} x_{1}^{2}+\frac{1}{2} x_{2}^{2}, x_{2} u \leq \frac{1}{2} x_{2}^{2}+\frac{1}{2} u^{2}, u^{2} \leq 32$ and $2 V^{2}(x) \leq x_{1}^{4}+x_{2}^{4} \quad$ (which hold for all $(x, u) \in \Re^{2} \times$ $[-4 \sqrt{2}, 4 \sqrt{2}])$, we obtain

$$
\begin{aligned}
\nabla V(x) f(x, u) & =g x_{1}^{2}-x_{1}^{4}+x_{1} x_{2}-x_{2}^{4}+x_{2} u \\
& \leq\left(g+\frac{1}{2}\right) x_{1}^{2}-x_{1}^{4}+x_{2}^{2}-x_{2}^{4}+\frac{1}{2} u^{2} \\
& \leq\left(g+\frac{1}{2}\right) x_{1}^{2}+x_{2}^{2}+16-2 V^{2}(x)
\end{aligned}
$$

for all $(x, u) \in \mathfrak{R}^{2} \times[-4 \sqrt{2}, 4 \sqrt{2}]$. Using (4.3), we get $\left(g+\frac{1}{2}\right) x_{1}^{2}+x_{2}^{2} \leq 2 V(x)$, which combined with the above inequality gives

$$
\nabla V(x) f(x, u) \leq 2 V(x)+16-2 V^{2}(x) .
$$

Inequality (4.4) shows that inequality (2.1) holds with $W(x):=\frac{1}{2} V(x)$ for all $(x, u) \in \mathfrak{R}^{2} \times[-4 \sqrt{2}, 4 \sqrt{2}]$ with $V(x) \geq R=4$.

We next show that assumption $\mathrm{H} 2$ holds with $P(x):=$ $\frac{1}{2} x_{1}^{2}+\frac{1}{2}\left(x_{2}+2 g x_{1}\right)^{2}, K_{1}:=\frac{1}{4}, \mu:=\frac{g}{4}$ and

$$
\begin{aligned}
k(x):=- & \left(5-\min \left(5, \max \left(4, \frac{1}{2} x_{1}^{2}+\frac{1}{2} x_{2}^{2}\right)\right)\right) \\
& \times \underset{U}{\operatorname{Pr}}\left(\left(4 g^{2}+1\right) x_{1}+3 g x_{2}+2 g\left(4 g^{2}-1\right) x_{1}^{3}\right. \\
& \left.+12 g^{2} x_{2} x_{1}^{2}+6 g x_{2}^{2} x_{1}\right)
\end{aligned}
$$

where $\operatorname{Pr}_{U}$ denotes the projection on the set $U=$ $[-4 \sqrt{2}, 4 \sqrt{2}] \subset \Re$. Note that since $V(x):=\frac{1}{2} x_{1}^{2}+\frac{1}{2} x_{2}^{2}$ we obtain the following estimate:

$$
\begin{aligned}
& \mid\left(4 g^{2}+1\right) x_{1}+3 g x_{2}+2 g\left(4 g^{2}-1\right) x_{1}^{3} \\
& \quad+12 g^{2} x_{2} x_{1}^{2}+6 g x_{2}^{2} x_{1} \mid \\
& \leq\left(4 g^{2}+3 g+1\right) \sqrt{2 V(x)}+2 g\left(\left|4 g^{2}-1\right|\right. \\
& \quad+6 g+3) 2 V(x) \sqrt{2 V(x)}
\end{aligned}
$$

for all $x \in \mathfrak{R}^{2}$. Taking into account (4.3) and the above inequality, we get

$$
\begin{aligned}
& \mid\left(4 g^{2}+1\right) x_{1}+3 g x_{2}+2 g\left(4 g^{2}-1\right) x_{1}^{3} \\
& \quad+12 g^{2} x_{2} x_{1}^{2}+6 g x_{2}^{2} x_{1} \mid \\
& \quad \leq 2\left(100 g^{2}+67 g+1\right) \sqrt{2} \leq 4 \sqrt{2}
\end{aligned}
$$

for all $x \in \mathfrak{R}^{2}$ with $V(x) \leq R=4$. Consequently, we obtain from (4.5) for all $x \in \mathfrak{R}^{2}$ with $V(x) \leq R=4$ :

$$
\begin{aligned}
& k(x)=-\left(\left(4 g^{2}+1\right) x_{1}+3 g x_{2}+2 g\left(4 g^{2}-1\right) x_{1}^{3}\right. \\
& \left.\quad+12 g^{2} x_{2} x_{1}^{2}+6 g x_{2}^{2} x_{1}\right)
\end{aligned}
$$


Using (4.6) and the definition $P(x):=\frac{1}{2} x_{1}^{2}+$ $\frac{1}{2}\left(x_{2}+2 g x_{1}\right)^{2}$, we obtain for all $x \in \Re^{2}$ with $V(x) \leq$ $R=4$ :

$$
\begin{aligned}
\nabla & P(x) f(x, k(x))=-g x_{1}^{2}-g\left(x_{2}+2 g x_{1}\right)^{2}-x_{1}^{4} \\
& +\left(x_{2}+2 g x_{1}\right)\left(-x_{2}^{3}+k(x)+\left(4 g^{2}+1\right) x_{1}\right. \\
& \left.-2 g x_{1}^{3}+3 g x_{2}\right) \\
= & -g x_{1}^{2}-g\left(x_{2}+2 g x_{1}\right)^{2}-x_{1}^{4}-\left(x_{2}+2 g x_{1}\right)^{4} \\
& +\left(x_{2}+2 g x_{1}\right)\left(k(x)+\left(4 g^{2}+1\right) x_{1}+3 g x_{2}\right. \\
& \left.+2 g\left(4 g^{2}-1\right) x_{1}^{3}+12 g^{2} x_{2} x_{1}^{2}+6 g x_{2}^{2} x_{1}\right) \\
= & -g x_{1}^{2}-g\left(x_{2}+2 g x_{1}\right)^{2}-x_{1}^{4}-\left(x_{2}+2 g x_{1}\right)^{4}
\end{aligned}
$$

Finally, the inequality

$$
\begin{aligned}
x_{1}^{2}+\left(x_{2}+2 g x_{1}\right)^{2} & \geq\left(1+4 g^{2}\right) x_{1}^{2}+x_{2}^{2}-4 g\left|x_{1} x_{2}\right| \\
& \geq\left(1-4 g^{2}\right) x_{1}^{2}+\frac{1}{2} x_{2}^{2}
\end{aligned}
$$

in conjunction with (4.3) implies the inequality

$$
x_{1}^{2}+\left(x_{2}+2 g x_{1}\right)^{2} \geq \frac{1}{2} x_{1}^{2}+\frac{1}{2} x_{2}^{2}
$$

for all $x \in \mathfrak{R}^{2}$. Inequality (4.8) in conjunction with (4.7) implies inequality (2.2) with $\mu:=\frac{g}{4}$. Moreover, inequality (4.8) in conjunction with the definition $P(x):=\frac{1}{2} x_{1}^{2}+$ $\frac{1}{2}\left(x_{2}+2 g x_{1}\right)^{2}$ implies the inequality $K_{1}|x|^{2} \leq P(x)$ with $K_{1}:=1 / 4$.

We next show that assumption $\mathrm{H} 3$ holds with

$$
\begin{aligned}
Q:= & \frac{1}{2}\left[\begin{array}{cc}
1 & -p \\
-p & 1
\end{array}\right], L:=-\frac{1}{2\left(1-p^{2}\right)} \\
& \times\left[\begin{array}{c}
2 g+2 p(1-p g)+4 p(11+2 \sqrt{7})^{2}+p \\
2 g p+4 p^{2}(11+2 \sqrt{7})^{2}+p^{2}+2(1-p g)
\end{array}\right] \\
\omega:= & \frac{p}{4}, b:=7
\end{aligned}
$$

where $p \in(0,1)$ is a constant. Indeed, by setting $\quad L_{1}=-\frac{2 g+2 p(1-p g)+4 p(11+2 \sqrt{7})^{2}+p}{2\left(1-p^{2}\right)}, \quad L_{2}=$ $-\frac{2 g p+4 p^{2}(11+2 \sqrt{7})^{2}+p^{2}+2(1-p g)}{2\left(1-p^{2}\right)}, \quad \tilde{e}=\left[\begin{array}{c}\tilde{e}_{1} \\ \tilde{e}_{2}\end{array}\right]=\left[\begin{array}{c}z_{1}-x_{1} \\ z_{2}-x_{2}\end{array}\right]$, we get

$$
\begin{aligned}
2(\tilde{e})^{\prime} & Q\left(f(z, u)-f(x, u)+L \tilde{e}_{1}\right) \\
= & g \tilde{e}_{1}^{2}-\tilde{e}_{1}\left(z_{1}^{3}-x_{1}^{3}\right)+\tilde{e}_{1} \tilde{e}_{2}+L_{1} \tilde{e}_{1}^{2} \\
& -p g \tilde{e}_{1} \tilde{e}_{2}-p \tilde{e}_{2}^{2}+p \tilde{e}_{1}\left(z_{1}^{3}-x_{1}^{3}\right)-p L_{1} \tilde{e}_{1} \tilde{e}_{2} \\
& +p \tilde{e}_{1}\left(z_{2}^{3}-x_{2}^{3}\right)-p L_{2} \tilde{e}_{1}^{2}-\tilde{e}_{2}\left(z_{2}^{3}-x_{2}^{3}\right)+L_{2} \tilde{e}_{1} \tilde{e}_{2} \\
= & \left(L_{1}+g-p L_{2}\right) \tilde{e}_{1}^{2}+\left(1-p g-p L_{1}+L_{2}\right) \tilde{e}_{1} \tilde{e}_{2} \\
& -p \tilde{e}_{2}^{2}-(1-p) \tilde{e}_{1}\left(z_{1}^{3}-x_{1}^{3}\right)-\tilde{e}_{2}\left(z_{2}^{3}-x_{2}^{3}\right) \\
& +p \tilde{e}_{1} \tilde{e}_{2}\left(z_{2}^{2}+x_{2} z_{2}+x_{2}^{2}\right) .
\end{aligned}
$$

Using the fact that $\tilde{e}_{1}\left(z_{1}^{3}-x_{1}^{3}\right) \geq 0, \tilde{e}_{2}\left(z_{2}^{3}-x_{2}^{3}\right) \geq 0$ and the above inequality, we obtain for all $u \in U, z, x \in \Re^{n}$ with $V(z) \leq 7$ and $V(x) \leq 4:$

$$
\begin{aligned}
& 2(\tilde{e})^{\prime} Q\left(f(z, u)-f(x, u)+L \tilde{e}_{1}\right) \leq\left(L_{1}+g-p L_{2}\right) \tilde{e}_{1}^{2} \\
& \quad+\left(1-p g-p L_{1}+L_{2}\right) \tilde{e}_{1} \tilde{e}_{2}-p \tilde{e}_{2}^{2} \\
& \quad+2 p\left|\tilde{e}_{1} \tilde{e}_{2}\right|(11+2 \sqrt{7}) .
\end{aligned}
$$

Completing the squares in the above inequality (i.e., using the inequality $2 p\left|\tilde{e}_{1} \tilde{e}_{2}\right|(11+2 \sqrt{7}) \leq \frac{p}{2} \tilde{e}_{2}^{2}+2 p(11+$ $2 \sqrt{7})^{2} \tilde{e}_{1}^{2}$ ), we obtain inequality (2.3) with $\omega:=\frac{p}{4}, b:=7$.

Finally, we show that assumption H4 holds with $a:=6$ and arbitrary $c \in(0,1)$, provided that

$$
\begin{aligned}
p & \leq 1 / 4 \text { and } p(597+176 \sqrt{7}) \\
& \leq \frac{123}{4 \sqrt{7}(\sqrt{7}+2)}-2-2 g .
\end{aligned}
$$

More specifically, we show next that the following inequality

$$
\nabla V(z)(f(z, u)+L(h(z)-h(x))) \leq-W(z)
$$

holds $u \in U, z, x \in \Re^{n}$ with $6<V(z) \leq 7$ and $V(x) \leq 4$. Using the definitions $V(x):=\frac{1}{2} x_{1}^{2}+\frac{1}{2} x_{2}^{2}, W(x):=\frac{1}{2} V(x)$ and the inequalities $z_{1} z_{2} \leq \frac{1}{2} z_{1}^{2}+\frac{1}{2} z_{2}^{2}, z_{2} u \leq \frac{1}{2} z_{2}^{2}+\frac{1}{2} u^{2}$, $u^{2} \leq 32$ and $2 V^{2}(z) \leq z_{1}^{4}+z_{2}^{4}$ (which hold for all $(z, u) \in$ $\Re^{2} \times[-4 \sqrt{2}, 4 \sqrt{2}]$ ), we conclude that inequality (4.11) holds provided that the following (more demanding) inequality

$$
\begin{aligned}
& \left(g+\frac{1}{2}\right) z_{1}^{2}+z_{2}^{2}+L_{1} z_{1}\left(z_{1}-x_{1}\right)+L_{2} z_{2}\left(z_{1}-x_{1}\right) \\
& +16 \leq-\frac{1}{2} V(z)+2 V^{2}(z)
\end{aligned}
$$

holds $u \in U, z, x \in \Re^{n}$ with $6<V(z) \leq 7$ and $V(x) \leq 4$. Using (4.3), we get $\left(g+\frac{1}{2}\right) z_{1}^{2}+z_{2}^{2} \leq 2 V(z)$, which implies that inequality (4.12) holds provided that the following (more demanding) inequality

$$
\frac{5}{2} V(z)+\left(\left|L_{1} z_{1}\right|+\left|L_{2} z_{2}\right|\right)\left|z_{1}-x_{1}\right|+16 \leq 2 V^{2}(z)
$$

holds $u \in U, z, x \in \Re^{n}$ with $6<V(z) \leq 7$ and $V(x) \leq 4$. Note that for all $z, x \in \Re^{n}$ with $6<V(z) \leq 7$ and $V(x) \leq$ 4 , it holds that $\left|z_{1}\right| \leq \sqrt{14},\left|z_{2}\right| \leq \sqrt{14},\left|z_{1}-x_{1}\right| \leq \sqrt{14}+$ $2 \sqrt{2}$. The previous inequalities imply that inequality (4.13) holds provided that the following inequality holds:

$$
\left|L_{1}\right|+\left|L_{2}\right| \leq \frac{41}{2 \sqrt{7}(\sqrt{7}+2)} .
$$


Since $\left|L_{1}\right|+\left|L_{2}\right|=(p+1) \frac{2 g+4 p(11+2 \sqrt{7})^{2}+p+2(1-p g)}{2\left(1-p^{2}\right)}$, it follows from (4.3) and (4.10) that inequality (4.14) holds.

Define

$$
\begin{aligned}
& \hat{k}(z, y, u):=L\left(z_{1}-y\right), \text { for all }(z, y, u) \in \mathfrak{R}^{2} \times \mathfrak{R} \\
& {[-4 \sqrt{2}, 4 \sqrt{2}] \text { with } z_{1}^{2}+z_{2}^{2} \leq 8,} \\
& \hat{k}(z, y, u):=L\left(z_{1}-y\right)-\frac{\varphi(z, y, u)}{|z|^{2}}\left[\begin{array}{c}
z_{1} \\
z_{2}
\end{array}\right], \\
& \quad \times \text { for all }(z, y, u) \in \mathfrak{R}^{2} \times \mathfrak{R} \times[-4 \sqrt{2}, 4 \sqrt{2}] \\
& \quad \text { with } z_{1}^{2}+z_{2}^{2}>8,
\end{aligned}
$$

where $\varphi: \Re^{2} \times \Re \times \Re \rightarrow \Re_{+}$is defined by

$$
\begin{aligned}
& \varphi(z, y, u):=\max \left(0,\left(g+\frac{1}{4}\right) z_{1}^{2}-z_{1}^{4}+z_{1} z_{2}-z_{2}^{4}\right. \\
& \left.+z_{2} u+\frac{1}{4} z_{2}^{2}+p\left(\frac{z_{1}^{2}+z_{2}^{2}}{2}\right)\left(L_{1} z_{1}+L_{2} z_{2}\right)\left(z_{1}-y\right)\right)
\end{aligned}
$$

and $p: \Re_{+} \rightarrow[0,1]$ is a locally Lipschitz function that satisfies $p(s)=1$ for all $s \geq b$ and $p(s)=0$ for all $s \leq a$. Let $q: \Re \rightarrow \Re_{+}$be the continuously differentiable function with $q(s):=2 s^{-1}-s^{-2}$ for $s>1$ and $q(s)=1$ for $s \leq 1$ with $s q(s) \leq K=2$ for $s \geq 1$. Let $\psi$ : $\Re^{n} \rightarrow[1,+\infty)$ be a smooth function defined by $\psi(z):=$ $\frac{1+2 \sqrt{14}}{2}+\frac{1}{2}|z|^{2}$, which satisfies implication (2.10) with $V(x):=\frac{1}{2} x_{1}^{2}+\frac{1}{2} x_{2}^{2}$ and $b=7$. Computing all constants involved in (2.11) and using all previous definitions, Theorem 2.2 implies that there exist a constant $\Gamma>0$ and a locally Lipschitz function $C \in K_{\infty}$ such that for every partition $\left\{\tau_{i}\right\}_{i=0}^{\infty}$ of $\Re_{+}$with $\sup _{i>0}\left(\tau_{i+1}-\tau_{i}\right) \leq T_{s}$, $v \in L^{\infty}\left(\Re_{+} ; \Re\right), \xi_{i, 0} \in L^{\infty}\left([-\delta, 0) ; \Re^{2}\right)(i=1, \ldots, N)$, $\left(z_{0}, w_{0}\right) \in \mathfrak{R}^{2} \times \mathfrak{R}, x_{0} \in C^{0}\left([-r, 0] ; \mathfrak{R}^{2}\right), u_{0} \in L^{\infty}([-r-$ $\tau, 0) ;[-4 \sqrt{2}, 4 \sqrt{2}])$, the solution of (4.1), (4.2) with

$$
\begin{gathered}
\dot{z}(t)=\left[\begin{array}{c}
g z_{1}(t)-z_{1}^{3}(t)+z_{2}(t) \\
-z_{2}^{3}(t)+u(t-r-\tau)
\end{array}\right]+\hat{k}(z(t), w(t), \\
u(t-r-\tau)), \text { for } t \geq 0
\end{gathered}
$$

$$
\dot{w}(t)=g z_{1}(t)-z_{1}^{3}(t)+z_{2}(t), \text { for } t \in\left[\tau_{i}, \tau_{i+1}\right), i \geq 0
$$

$$
w\left(\tau_{i}\right)=y\left(\tau_{i}\right), \text { for } i \geq 1
$$

$$
\begin{aligned}
\xi_{j}(t)= & q\left(\frac{\left|\xi_{j-1}(t)\right|}{\psi(z(t))}\right) \xi_{j-1}(t)+\int_{0}^{\delta} f\left(q\left(\frac{\left|\xi_{j}(t+s-\delta)\right|}{\psi(z(t))}\right)\right. \\
& \left.\times \xi_{j}(t+s-\delta), u(t+(j-1) \delta-\tau-r+s)\right) d s \\
& \text { for } t \geq 0, j=1, \ldots, N
\end{aligned}
$$

with $\xi_{0}(t)=z(t)$ and

$$
u(t)=k\left(\xi_{N}(t)\right), \text { for } t \geq 0
$$

initial condition $\xi_{j}(\theta)=\xi_{j, 0}(\theta)$ for $\theta \in[-\delta, 0) \quad(j=$ $1, \ldots, N),(z(0), w(0))=\left(z_{0}, w_{0}\right), x(\theta)=x_{0}(\theta)$ for $\theta \in$ $[-r, 0], u(\theta)=u_{0}(\theta)$ for $\theta \in[-r-\tau, 0)$, exists and satisfies estimate (2.17) for all $t \geq 0$, provided that $\delta=\frac{r+\tau}{N}$, $N>0$ is an integer and $T_{s}>0, \sigma>0$ are constants so that

$$
\begin{aligned}
\sigma & <\min \left(\frac{g}{8\left(1+2 g^{2}\right) \sqrt{2}}, \frac{p}{8(1+p)}\right), \delta \\
e^{\sigma \delta} & <\frac{27(1+2 \sqrt{14})}{(27+54 \sqrt{14}+32 \sqrt{2})(3034+g)} \text { and } \\
T_{s} e^{\sigma T_{s}} & <\frac{\sqrt{2} p \sqrt{1-p}}{12(43+g)|L|(1+p)^{3 / 2}} .
\end{aligned}
$$

Of course, it should be noted that inequalities (4.23) are highly conservative. The control practitioner can use inequalities (4.23) only as a first step for the selection of the parameters. The next step is the determination of the optimal values of the parameters by means of extensive numerical experiments.

Example 4.2: Consider the nonlinear system

$$
\begin{aligned}
\dot{x}_{1}(t) & =g x_{1}(t)-x_{1}^{3}(t)+x_{2}(t), \\
\dot{x}_{2}(t) & =-x_{2}^{3}(t)+p\left(x_{3}(t)\right)+v_{1}(t-\tau), \\
\dot{x}_{3}(t) & =v_{2}(t-\tau), \\
x(t) & =\left(x_{1}(t), x_{2}(t), x_{3}(t)\right) \in \mathfrak{R}^{3}, \quad v(t) \in \mathfrak{R}^{2},
\end{aligned}
$$

where $\tau>0$ is the input delay, $p \in C^{\infty}(\Re ; \Re)$ is a smooth function with $p(0)=0$ and $g>0$ is a constant. The measured output is given by the equation:

$$
y\left(\tau_{i}\right)=\left[\begin{array}{l}
y_{1}\left(\tau_{i}\right) \\
y_{2}\left(\tau_{i}\right)
\end{array}\right]=\left[\begin{array}{l}
x_{1}\left(\tau_{i}-r\right) \\
x_{3}\left(\tau_{i}-r\right)
\end{array}\right],
$$

where $\left\{\tau_{i}\right\}_{i=0}^{\infty}$ is the sampling partition (a partition of $\Re_{+}$) and $r \geq 0$ is the measurement delay. All assumptions of Theorem 2.4 are satisfied with

$$
\begin{gathered}
a_{1}(x):=x_{3}, l=1 a_{2}(\theta)=\left[\begin{array}{c}
-p(\theta) \\
-\theta
\end{array}\right] g(\theta, v)=v_{2} \Phi(y, v) \\
:=y_{2}+\int_{-r-\tau}^{0} v_{2}(s) d s U=[-4 \sqrt{2}, 4 \sqrt{2}] \times\{0\}
\end{gathered}
$$

under the assumption that inequality (4.3) holds. Indeed, notice that after an initial transient period system (4.24) with 


$$
\begin{gathered}
v(t)=u(t)-\left[\begin{array}{c}
p(\theta(t)) \\
\theta(t)
\end{array}\right], \text { for } t \geq 0 \\
\dot{\theta}(t)=-\theta(t)+u_{2}(t), \text { for } t \in\left[\tau_{i}, \tau_{i+1}\right) i \geq 0 \\
\theta\left(\tau_{i}\right)=y_{2}\left(\tau_{i}\right)+\int_{\tau_{i}-r-\tau}^{\tau_{i}} v_{2}(s) d s, \text { for } i \geq 1
\end{gathered}
$$

is transformed to system (4.1) with the additional equation:

$$
\dot{x}_{3}(t)=-x_{3}(t)+u_{2}(t-\tau) .
$$

It follows from Theorem 2.4 that for every partition $\left\{\tau_{i}\right\}_{i=0}^{\infty}$ of $\Re_{+}$with $\sup _{i \geq 0}\left(\tau_{i+1}-\tau_{i}\right) \leq T_{s}$, the solution of the closed-loop system (4.24) with (4.27)-(4.29), (4.18)(4.20), (4.21) and (4.22) with $\xi_{0}(t)=z(t)$ satisfies $(2.25)$ provided that $\delta=\frac{r+\tau}{N}, N>0$ is an integer and $T_{s}>0$, $\sigma>0$ are constants so that (4.23) holds. A closer inspection of the closed-loop system (4.24) with (4.27)-(4.29), (4.18)-(4.20), (4.21), (4.22) and $\xi_{0}(t)=z(t)$ reveals that not only the exponential attractivity property holds for the closed-loop system but also the properties of Lagrange and Lyapunov stability (see (Karafyllis \& Jiang, 2011)).

\section{Concluding remarks}

In this work, we have shown that the ISP-O-P-DFC control scheme can be applied to nonlinear systems with a compact absorbing set. The results guarantee the following properties for the closed-loop system even when the full state is not measured and when the measurement is sampled and possibly delayed:

- Global asymptotic stability and exponential convergence for the disturbance-free case;

- Robustness with respect to perturbations of the sampling schedule;

- Robustness with respect to measurement errors.

The input and measurement delays can be arbitrarily large but must be both accurately known and constant.

To our knowledge, this is the first time that predictor feedback is proposed for systems with a compact absorbing set. While predictor feedback has been proposed and applied to nonlinear systems for which the solution mapping is known (see Karafyllis \& Krstic, 2012a; Krstic, 2010), or to disturbance-free systems with state measurements (see Karafyllis \& Krstic, 2012b), here we are in a position to guarantee important properties for the closed-loop system under the effect of various disturbances and without knowledge of the solution mapping. The results of the present work can be directly compared to the results in Karafyllis and Krstic (2013a) for globally Lipschitz systems. However, in this paper, we have not considered the effect of modelling errors as in Karafyllis and Krstic (2013a): this is a topic for future research.

More remains to be done for the class of systems that can be transformed to a nonlinear system with a compact absorbing set by means of a preliminary predictor feedback. Although Theorem 2.4 guarantees global exponential attractivity in the absence of measurement errors, additional assumptions must be employed for the global asymptotic stability and exponential convergence in the disturbancefree case.

An extension of the previous results to the case where the control is applied through a zero-order hold device is also an open problem and it is under investigation by the authors.

\section{References}

Ahmed-Ali, T., Karafyllis, I., \& Lamnabhi-Lagarrigue, F. (2013). Global exponential sampled-data observers for nonlinear systems with delayed measurements. Systems and Control Letters, 62(7), 539-549.

Angeli, D., \& Sontag, E.D. (1999). Forward completeness, unbounded observability and their Lyapunov characterizations. Systems and Control Letters, 38(4-5), 209-217.

Bekiaris-Liberis, N., \& Krstic, M. (2012). Compensation of timevarying input and state delays for nonlinear systems. Journal of Dynamic Systems, Measurement, and Control, 134(1), doi: $10.1115 / 1.4005278$

Bekiaris-Liberis, N., \& Krstic, M. (2013a). Compensation of statedependent input delay for nonlinear systems. IEEE Transactions on Automatic Control, 58, 275-289.

Bekiaris-Liberis, N., \& Krstic, M. (2013b). Robustness of nonlinear predictor feedback laws to time- and state-dependent delay perturbations. Automatica, 49(6), 1576-1590.

Germani, A., Manes, C., \& Pepe, P. (2002). A new approach to state observation of nonlinear systems with delayed output. IEEE Transactions on Automatic Control, 47(1), 96101.

Karafyllis, I. (2011). Stabilization by means of approximate predictors for systems with delayed input. SIAM Journal on Control and Optimization, 49(3), 1100-1123.

Karafyllis, I., \& Jiang, Z.-P. (2011). Stability and stabilization of nonlinear systems (Series: Communications and Control Engineering). London: Springer-Verlag.

Karafyllis, I., \& Krstic, M. (2012a). Nonlinear stabilization under sampled and delayed measurements, and with inputs subject to delay and zero-order hold. IEEE Transactions on Automatic Control, 57(5), 1141-1154.

Karafyllis, I., \& Krstic, M. (2012b). Numerical schemes for nonlinear predictor feedback. Mathematics of Control, Signals and Systems (see also arXiv:1211.1121 [math.OC]). Manuscript submitted for publication.

Karafyllis, I., \& Krstic, M. (2013a). Stabilization of nonlinear delay systems using approximate predictors and high-gain observers. Automatica, 49(12), 3623-3631.

Karafyllis, I., \& Krstic, M. (2013b). On the relation of delay equations to first-order hyperbolic partial differential equations. ESAIM Control, Optimisation and Calculus of Variations (see also arXiv:1302.1128 [math.OC]). Manuscript submitted for publication.

Khalil, H.K. (1996). Nonlinear systems (2nd ed.). Upper Saddle River, NJ: Prentice-Hall. 
Krstic, M. (2004). Feedback linearizability and explicit integrator forwarding controllers for classes of feedforward systems. IEEE Transactions on Automatic Control, 49(10), 16681682.

Krstic, M. (2008). Lyapunov tools for predictor feedbacks for delay systems: Inverse optimality and robustness to delay mismatch. Automatica, 44(11), 2930-2935.

Krstic, M. (2009). Delay compensation for nonlinear, adaptive, and PDE systems. Boston, MA: Birkhäuser.

Krstic, M. (2010). Input delay compensation for forward complete and strict-feedforward nonlinear systems. IEEE Transactions on Automatic Control, 55(2), 287-303.
Mazenc, F., Malisoff, M., \& Lin, Z. (2008). Further results on input-to-state stability for nonlinear systems with delayed feedbacks. Automatica, 44(9), 2415-2421.

Mazenc, F., Mondie, S., \& Francisco, R. (2004). Global asymptotic stabilization of feedforward systems with delay at the input. IEEE Transactions on Automatic Control, 49(5), 844850.

Stuart, A.M., \& Humphries, A.R. (1998). Dynamical systems and numerical analysis. Cambridge: Cambridge University Press.

Temam, R. (1997). Infinite-dimensional dynamical systems in mechanics and physics (2nd ed.). New York, NY: SpringerVerlag. 